\title{
Bird Species Abundance and Their Correlationship with Microclimate and Habitat Variables at Natural Wetland Reserve, Peninsular Malaysia
}

\author{
Muhammad Nawaz Rajpar ${ }^{1}$ and Mohamed Zakaria ${ }^{2}$ \\ ${ }^{1}$ Forest Education Division, Pakistan Forest Institute, Peshawar 25120, Pakistan \\ ${ }^{2}$ Faculty of Forestry, Universiti Putra Malaysia (UPM), Selangor, 43400 Serdang, Malaysia \\ Correspondence should be addressed to Mohamed Zakaria, mzakaria@putra.upm.edu.my
}

Received 6 May 2011; Revised 29 August 2011; Accepted 5 September 2011

Academic Editor: Iain J. McGaw

Copyright (C) 2011 M. N. Rajpar and M. Zakaria. This is an open access article distributed under the Creative Commons Attribution License, which permits unrestricted use, distribution, and reproduction in any medium, provided the original work is properly cited.

\begin{abstract}
Birds are the most conspicuous and significant component of freshwater wetland ecosystem. Presence or absence of birds may indicate the ecological conditions of the wetland area. The objectives of this study were to determine bird species abundance and their relationship with microclimate and habitat variables. Distance sampling point count method was applied for determining species abundance and multiple regressions was used for finding relationship between bird species abundance, microclimate and habitat variables. Bird species were monitored during November, 2007 to January, 2009. A total of 8728 individual birds comprising 89 species and 38 families were detected. Marsh Swamp was swarmed by 84 species $(69.8 \%)$ followed open water body by 55 species (17.7\%) and lotus swamp by 57 species (12.6\%). Purple swamphen Porphyrio porphyrio (9.1\% of all detections) was the most abundant bird species of marsh swamp, lesser whistling duck-Dendrocygna javanica (2.3\%) was dominant species of open water body and pink-necked green pigeon-Treron vernans (1.7\%) was most common species of lotus swamp. Results revealed that the habitat characteristics such as vegetation composition (i.e. emergent and submerged vegetations, grasses, shrubs, and trees), vegetation structures (tree diameter and height) and microclimate variables (temperature, relative humidity and light intensity) were the key factors that influenced the distribution, diversity and density of the wetland bird species. This study also revealed that the wetland bird species have adapted a fairly unique set of microhabitat and microclimate conditions.
\end{abstract}

\section{Introduction}

Wetlands are characterized by shallow water overlying waterlogged soil, interspersed submerged and emergent vegetation [1-3]. Wetlands are the most productive ecosystems due to their habitat diversity, great productivity, and diverse attributes including a distinctive avifauna. During the past century, wetland areas have been reduced by more than $50 \%$ globally, and their destruction will likely continue [4-6]. The gradual loss and degradation of wetlands due to development [7] and pollution [8] have also been adversely affected wetland bird species [9-11].

Birds are an important component of biotic community of wetland ecosystems [12]. They respond quickly to changing in habitat $[13,14]$. Thus, birds are good bioindicators of wetland habitat quality, productivity, and stability. Birds often have correlation with their habitats [15] and have also been used as surrogates for assessing the impact of habitat changes $[16,17]$. Monitoring the species abundance, habitat preference, and correlationship between species abundance and habitat provides basic information for determining factors causing population fluctuation of bird species [18]. Subsequently, the information helps in conservation and management of threatened and endangered species [19]. Long-term avian monitoring identifies the bird species that decline due to habitat loss or degradation. Similarly, the assessment of vegetation composition and structure is a useful tool to examine and understand the habitat characteristics and impacts of disturbance or alteration of habitats on the avian species. The alteration in wetland habitats may cause changes in avian abundance and diversity [20]. 
Distance sampling point count method is widely used to examine the avian species abundance in different habitats and the association of bird species with habitats characteristics and microclimates [21-23]. This method involves the visual and auditory detection of birds within fixed- or variable-radius plots [24]. The relative abundance of bird species is often associated with vegetation community and food resources and positively correlated with habitat structural complexity $[25,26]$.

Malaysia is blessed with extensive wetland areas, that is, 3.5 to 4.0 million ha or $10.0 \%$ of the total land area. This total wetland area is divided into marshes, nipa swamps, mangrove, mudflats, freshwater swamps, peat swamp forests, lakes, rivers, sandy beaches, and rocky shore. Information about relative abundance of birds, habitat preference, and association with habitat characteristics and microclimate variables for various wetlands is lacking [27]. Long-term population trends of wetland birds, habitat and microclimate characteristics, as well as correlationship between wetland bird species and habitat characteristics have not been studied. In fact, very little is known on the ecological roles of bird species in relation to habitat and habitat disturbances. Hence, it is important to determine the relative abundance, habitat preference, and correlationship of bird species between habitat characteristics and microclimate variables.

\section{Materials and Methods}

2.1. Study Site. Paya Indah (a Malay translation of "beautiful swamp") Wetland Reserve encompasses 3050 ha of lands, out of which 450 ha are under the administrative control of the Department of Wildlife and National Parks, Peninsular Malaysia. Presently study area is located within the quadrant of $101^{\circ} 10^{\prime}$ to $101^{\circ} 50^{\prime}$ longitude and $2^{\circ} 50^{\prime}$ and $3^{\circ} 00^{\prime}$ latitude (Figure 1). A distinctive feature of this natural wetland area is that it comprises of three major habitats, that is, marsh swamp, lotus swamp and open water body that may vary due to heterogeneity of the existing vegetation composition, vegetation structure, hydrology, and productivity and represent specific environmental features that satisfy the biological needs of wetland bird species.

2.2. Marsh Swamp. Marsh swamp was shallow water dominated by lush growths of aquatic vegetation such as sedges, reeds, rushes, and grasses. The plants grow with their stems partly in and partly out of the water. About $85 \%$ of the total marsh swamp areas are covered with water, while $15 \%$ are terrestrial [28]. The marsh swamp was predominantly covered with aquatic plants, namely, Eleocharis dulcis, Lepironia articulata, Stenochlaena palustris, Philydrum lanuginosum, and Scleria purpurascens, and scattered trees such as Acacia auriculiformis, A. mangium, Macaranga tanarius, Peltophorum pterocarpum, Cinnamomum iners, Melicope glabra, and Melastoma malabathricum along the edges. This area was shallow in water depth and rich in prey resources, that is, fish, amphibians, insects, snails, and larvae of invertebrates.

2.3. Lotus Swamp. Lotus swamp was shallow water body dominated by water lilies, sedges, reeds, and grasses. It allows plants to grow and reach the water surface. About $90 \%$ of the lotus swamp areas are covered by water, whereas $10 \%$ are terrestrial [28]. This area was extensively covered with Nelumbo nucifera, N. nouchali, N. pubescens, E. dulcis, Elodea sp., Phragmites karka reeds, and Typha angustifolia. On the contrary, the dry land is covered with A. auriculiformis, A. mangium, and some parts with $M$. malabathricum.

2.4. Open Water Body. Open water body was a larger and deep area dominated by submerged vegetation while the edges in shallow water were covered by reeds, sedges, and grasses. About $90 \%$ of the total area was covered by water and $10 \%$ by dryland [28]. Open Water Bodies was mostly covered with emergent vegetation, such as Nymphaea odorata, Potamogeton spp., E. dulcis, Myriophyllum spicatum, Salvinia molesta, Scirpus sylvaticus, S. californicus, S. mucronatus, and S. maritimus. The edges were dominated by E. dulcis, $S$. purpurascens, Sagittaria latifolia, and Hydrilla sp. Open water bodies are rich in invertebrates, amphibians, and fish.

2.5. Bird Surveys. Bird species relative abundance was counted using distance sampling from count points from November 2007 to January 2009. One hundred and twentyone point count stations at $300 \mathrm{~m}$ interval were established within three habitats, namely, Marsh Swamp (43 stations), Lotus Swamp (38 stations), and Open Water Body (40 stations), to avoid double counting of the same birds at more than one station. The birds were counted 15 consecutive times at a monthly interval for each station in order to obtain reliable estimates and reduce bias. The survey was conducted early in the morning from 0730 to $1100 \mathrm{hrs}$. Birds were counted at each station for 10 minutes following Gutzwiller [29], Jimenez [30], Lee and Marsden [31], and Zakaria et al. [28]. During each count, all bird species and individuals seen or heard were recorded. Flushed birds were also recorded, and their known original positions were included in the analysis. However, flying birds were not recorded due to unknown original position. The methodology was used as described by Bibby et al. [32], Buckland et al. [33], Aborn [34] and Nadeau et al. [35].

2.6. Vegetation Assessment. The vegetation variables were sampled in three habitats using the quadrat method $(10 \mathrm{~m} \times$ $10 \mathrm{~m}$ ) simultaneously at the sites where birds were counted. This method is one of the most commonly used and accepted methods to survey vegetation in a variety of habitats $[8,36$, 37]. A total of 120 quadrant plots were sampled for vegetation structure within the consistency of the point count stations. In each sample plot, soil cover percent (i.e., the proportion of soil surface covered with vegetation and bare one \%), species richness (i.e., the number of plant species), vegetation type (i.e., trees, shrubs, grasses, emerged and submerged vegetation, sedges, reeds, ferns, and herbs), vegetation height (i.e., meters), vegetation diameter (i.e., centimetres), and microclimate, such as temperature, relative humidity, and light intensity, were recorded. The soil cover, proportion of shrubs, grasses, emerged and submerged vegetation, sedges, reeds, ferns, and herbs were recorded using the visual 


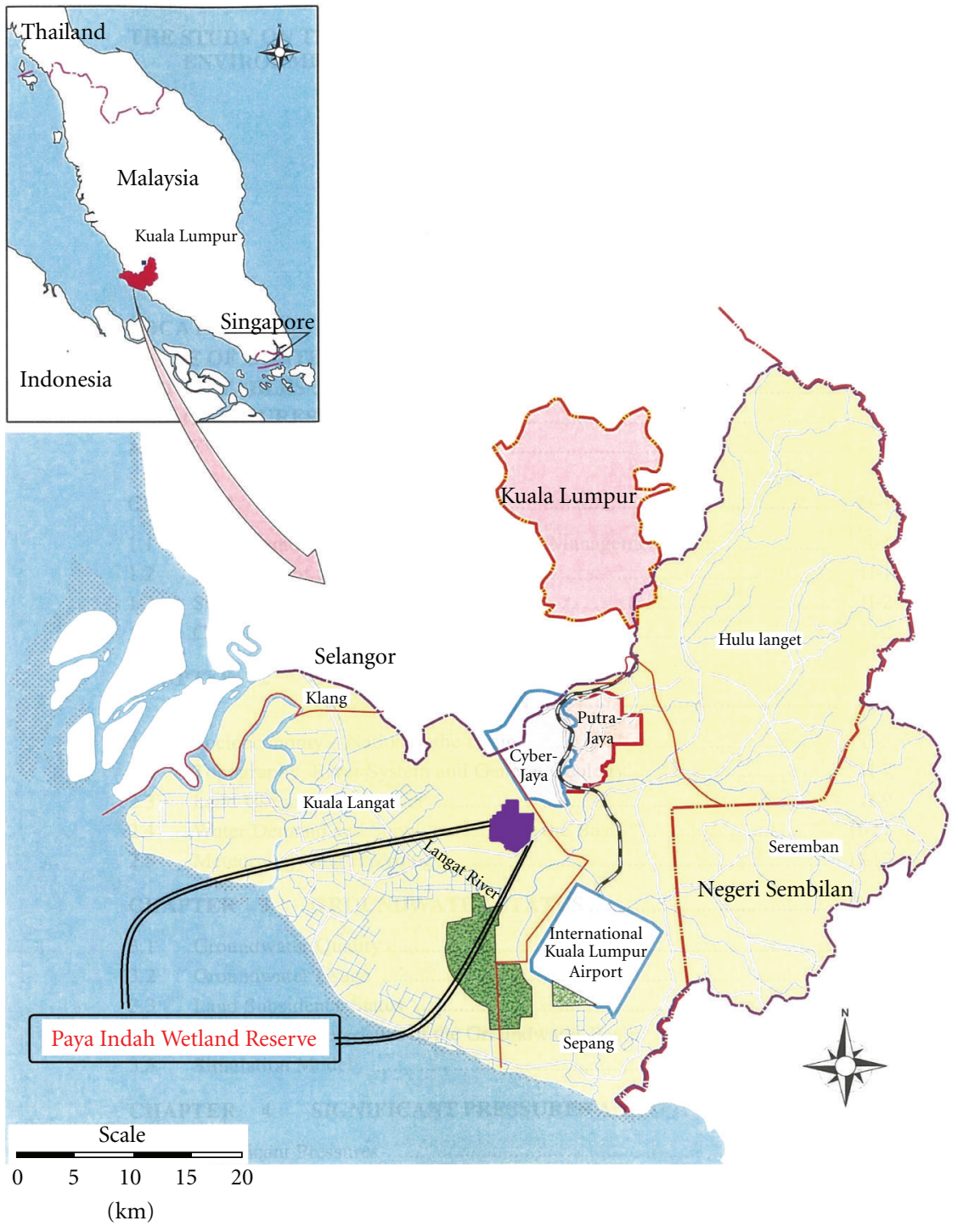

Legend

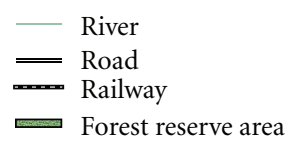

$$
\begin{array}{ll}
\ldots & \text { State boundary } \\
- & \text { District boundary } \\
-.-= & \text { Boundary of langat } \\
& \text { basin (study area) }
\end{array}
$$

Figure 1: Location map of the Paya Indah Wetland Reserve, Peninsular Malaysia.

estimation while the temperature and relative humidity were recorded using psychrometer. In addition, light intensity was recorded using the LUX meter. The methodology was according to Isacch et al. [38].

Plant samples were brought from the field and processed for plant pressure. The pressed plant samples were kept in the oven for drying for one week. Plants were identified using field guides and cross-checked with the samples kept in the Herbarium of the Faculty of Forestry, Universiti Putra Malaysia, Malaysia.

2.7. Analysis. The relative abundance (\%) of bird species was estimated using the following expression: $n / N \times 100$ [28], where $n$ is the number of a particular bird species and $N$ is the total observations detected for all species. The correlationship of birds, microclimate variables, and habitat characteristics was determined using multiple regressions of Constrained Redundancy Ordination (RDA) of canonical correspondence analysis (CCA) Software Version 4.5 by Ter Braak and Smilauer [39] in order to understand the variable factors that influenced distribution of bird species in the study area. Canonical correlation analysis (CCA) is a way to determine the linear relationship between two multidimensional variables. It has two bases, in which the correlation matrix between the variables is diagonal and the correlations on the diagonal are maximized. The advantage of canonical 
correlation is that, it is invariant with respect to affine transformations of the variables.

Let $x=\left(x^{1}, \ldots, x^{n}\right)$ denote $n$ observations of an $m$ dimensional random vector, let $x j=\left(x^{J} 1, \ldots, x^{J} m\right)$ denote the $j$ th observation.

The sample means $\tilde{\mu}$ and sample covariance matrix $\tilde{\Sigma}$ are defined as

$$
\tilde{\mu}=\frac{1}{n} \sum_{j=1}^{n} x^{j}, \quad \tilde{\Sigma}=\frac{1}{n} \sum_{j=1}^{n}\left(x^{j}-\tilde{\mu}\right)\left(x^{j}-\tilde{\mu}\right)^{\top} .
$$

The relative abundance data was used for correlation analysis of three habitats instead of the density data. The relative abundance data was used firstly to maximize the correlation of most bird species with microclimate variables and habitat characteristics. Secondly, most of the bird species in each habitat had very low detections ( $<5$ individuals).

\section{Results}

During the study period, a total of 8728 individual birds, comprising of 89 species and 38 families, were recorded.

3.1. Relative Abundance of Birds in Marsh Swamp. In Marsh Swamp, a total of 6,086 bird observations, that is, $69.7 \%$ of all detections, were recorded. The birds belong to 84 species and 37 families. Three species, purple swamphen-Porphyrio porphyrio (9.1\% of all detections), yellow-vented bulbul-Pycnonotus goiavier (7.9\%), and pink-necked green pigeon-Treron vernans $(7.0 \%)$ count showed the highest relative abundance. On the contrary, nine bird species, ashy drongo-Dicrurus leucophaeus, black baza-Aviceda leuphotes, blue-breasted quail-Coturnix chinensis, house crowCorvus splendens, inornate warbler-Phylloscopus inornatus, little spiderhunter-Arachnothera longirostra, pheasant-tailed jacana-Hydrophasianus chirurgus, rusty-rumped warblerLocustella certhiola, and speckled piculet-Picumnus innominatus showed the least relative abundance, observed only once $(0.01 \%$ each $)$ in the wetland reserve (Table 1$)$.

3.2. Relative Abundance of Birds in Lotus Swamp Habitat. A total of 1,097 bird observations (12.6\%) were recorded in Lotus Swamp. The birds belong to 57 species and 30 families. Pink-necked green Pigeon-Treron vernans (1.7\%), yellowvented bulbul-Pycnonotus goiavier and peaceful doveGeopelia striata (1.2\% each) were the three most common birds in the lotus swamp habitat. Whereas, 12 bird species, Richard's Pipit-Anthus richardi, oriental reed warbler-Acrocephalus orientalis, olive-backed sunbird-Nectarinia jugularis, cotton pygmy goose-Nettapus coromandelianus, little green pigeon-Treron olax, barred button quail-Turnix suscitator, water cock-Gallicerx cinerea, common kingfisherAlcedo atthis, long-tailed shrike-Lanius schach, little spiderhunter-Arachnothera longirostra, rusty-rumped warblerLocustella certhiola, copper-throated sunbird-Nectarinia calcostetha and thick-billed green pigeon-Treron curvirostra, were the least common in lotus swamp habitat, recorded only once $(0.01 \%$ each) (Table 1$)$.
3.3. Relative Abundance of Birds in Open Water Body. In open water body habitat a total of 1,545 bird observations $(17.7 \%)$ of all detections were recorded that belong to 55 bird species and 33 families. The results indicated that lesser whistling duck-Dendrocygna javanica (2.3\%), blue-tailed bee-eater-Merops philippinus (1.6\%) and yellow-vented bulbul-Pycnonotus goiavier (1.5\%) were the three most dominant bird species. In contrast, eight bird species, Eurasian tree sparrow-Passer montanus, grey heron-Ardea cinerea, oriental reed warbler-Acrocephalus orientalis, Schrenck's bittern-Ixobrychus eurhythmus, mangrove whistlerPachycephala grisola, Savanna nightjar-Caprimulgus affinis, large-tailed nightjar-Caprimulgus macrurus, and black baza-Aviceda leuphotes, were the rarest birds, recorded only once $(0.01 \%$ each) (Table 1$)$.

3.4. Microclimate and Habitat Variables of Marsh Swamp. Microclimate data indicated that the marsh swamp had $28.1^{\circ} \mathrm{C}$ mean temperature $\left(25-31^{\circ} \mathrm{C}\right), 95.3 \%$ mean relative humidity (89-97\%), and mean 233.65 Lux light intensity (16-520 Lux). The habitat variables showed that marsh swamp area was covered by emergent vegetation $(59.1 \%)$, submerged vegetation (13.6\%), grasses (4.8\%), and shrubs $(3.7 \%)$, while $18.7 \%$ of the land was barren. In addition, 67 tree species were recorded along the lake edges in the marsh swamp area (Table 2).

3.5. Microclimate and Habitat Variables of Lotus Swamp. The lotus swamp had $27.5^{\circ} \mathrm{C}$ mean temperature $\left(26-30^{\circ} \mathrm{C}\right)$, $96.4 \%$ mean relative humidity (94-97\%), and mean 260.60 Lux light intensity (150-362 Lux). On the other hand, the habitat variables indicated that $77.0 \%$ of the lotus swamp habitat was covered with vegetation, that is, emergent vegetation $(55.4 \%)$, submerged vegetation $(15.4 \%)$, grasses $(5.0 \%)$, and shrubs $(1.2 \%)$. In addition, $23.0 \%$ of the area was bare land and walking paths. There were 17 tree species in the lotus swamp habitat (Table 2).

3.6. Microclimate and Microhabitat Variables of Open Water Body. Microclimate data indicated that the open water body habitat had $28.5^{\circ} \mathrm{C}$ mean temperature $\left(26.5-30^{\circ} \mathrm{C}\right), 95.5 \%$ mean relative humidity (94-97\%), and mean 438.16 Lux light intensity (351-517 Lux). Microhabitat variables showed $76.7 \%$ of the area was covered with vegetation, while the rest was dry land area including walking paths. Out of $76.7 \%$ of the total vegetated area, $61.5 \%$ was dominated by submerged vegetation, $30.8 \%$ by emergent vegetation, $4.5 \%$ by grasses, and $3.2 \%$ by shrubs. Besides, there were six tree species in open water body (Table 2 ).

3.7. Correlation of Birds, Microclimate, and Microhabitat in Marsh Swamp. The RDA ordination biplot diagram of marsh swamp habitat indicated that cotton pygmy geese and lesser whistling ducks had a strong association with the submerged vegetation. In addition, white-browed crakes, yellow bitterns, purple herons also showed a positive correlationship with the submerged vegetation. Schrenck's bitterns, common moorhens, purple swamphens, black-headed munias and white-headed munias indicated strong positive association 
TABLE 1: Relative abundance of bird species recorded at three habitats of Paya Indah Wetland Reserve, Peninsular Malaysia.

\begin{tabular}{|c|c|c|c|c|c|c|c|c|}
\hline \multirow{3}{*}{ Family name } & \multirow{3}{*}{ Scientific name } & \multirow{3}{*}{ Common name } & \multicolumn{6}{|c|}{ No. of observations with habitat } \\
\hline & & & \multicolumn{2}{|c|}{ Marsh swamp } & \multicolumn{2}{|c|}{ Lotus swamp } & \multicolumn{2}{|c|}{ Open water body } \\
\hline & & & Observation & $\begin{array}{l}\% \text { of all } \\
\text { detection }\end{array}$ & Observation & $\begin{array}{l}\% \text { of all } \\
\text { detection }\end{array}$ & Observation & $\begin{array}{c}\% \text { of all } \\
\text { detection }\end{array}$ \\
\hline Rallidae & $\begin{array}{l}\text { Porphyrio } \\
\text { porphyrio }\end{array}$ & $\begin{array}{l}\text { Purple } \\
\text { swamphen }\end{array}$ & 798 & 9.14 & 78 & 0.89 & 25 & 0.29 \\
\hline Pycnonotidae & Pycnonotus goiavier & $\begin{array}{l}\text { Yellow-vented } \\
\text { bulbul }\end{array}$ & 690 & 7.91 & 101 & 1.16 & 129 & 1.48 \\
\hline Columbidae & Treron vernans & $\begin{array}{l}\text { Pink-necked } \\
\text { green pigeon }\end{array}$ & 614 & 7.03 & 150 & 1.72 & 90 & 1.03 \\
\hline Columbidae & Geopelia striata & Peaceful dove & 462 & 5.29 & 101 & 1.16 & 84 & 0.96 \\
\hline Columbidae & $\begin{array}{l}\text { Streptopelia } \\
\text { chinensis }\end{array}$ & Spotted dove & 386 & 4.42 & 56 & 0.64 & 67 & 0.77 \\
\hline Hirundinidae & Hirundo tahitica & Pacific swallow & 208 & 2.38 & 39 & 0.45 & 85 & 0.97 \\
\hline Rallidae & $\begin{array}{l}\text { Amaurornis } \\
\text { phoenicurus }\end{array}$ & $\begin{array}{l}\text { White-breasted } \\
\text { waterhen }\end{array}$ & 200 & 2.29 & 38 & 0.44 & 25 & 0.29 \\
\hline Ploceidae & Ploceus philippinus & Baya weaver & 173 & 1.98 & 7 & 0.08 & 52 & 0.60 \\
\hline Sturnidae & Acridotheres tristis & Common myna & 166 & 1.90 & 17 & 0.19 & 51 & 0.58 \\
\hline Ardeidae & Ardea purpurea & Purple heron & 164 & 1.88 & 52 & 0.60 & 22 & 0.25 \\
\hline Ardeidae & Ixobrychus sinensis & Yellow bittern & 162 & 1.86 & 42 & 0.48 & 11 & 0.13 \\
\hline Sturnidae & Acridotheres fuscus & Jungle myna & 154 & 1.76 & 15 & 0.17 & 117 & 1.34 \\
\hline Alcedinidae & Halcyon smyrnensis & $\begin{array}{l}\text { White-throated } \\
\text { kingfisher }\end{array}$ & 128 & 1.47 & 51 & 0.58 & 42 & .48 \\
\hline Estrildidae & $\begin{array}{l}\text { Lonchura } \\
\text { punctulata }\end{array}$ & $\begin{array}{l}\text { Scaly-breasted } \\
\text { munia }\end{array}$ & 125 & 1.43 & 36 & 0.41 & 49 & 0.56 \\
\hline Estrildidae & Lonchura malacca & $\begin{array}{l}\text { Black-headed } \\
\text { munia }\end{array}$ & 122 & 1.40 & 0 & 0 & 3 & 0.03 \\
\hline Sturnidae & Aplonis panayensis & $\begin{array}{l}\text { Philippine } \\
\text { glossy starling }\end{array}$ & 116 & 1.33 & 0 & 0 & 24 & 0.27 \\
\hline Motacillidae & Anthus richardi & Richard's pipit & 114 & 1.31 & 1 & 0.01 & 26 & 0.30 \\
\hline Passeridae & Passer montanus & $\begin{array}{l}\text { Eurasian tree } \\
\text { sparrow }\end{array}$ & 94 & 1.08 & 8 & 0.09 & 1 & 0.01 \\
\hline Charadriidae & Vanellus indicus & $\begin{array}{l}\text { Red-wattled } \\
\text { lapwing }\end{array}$ & 93 & 1.07 & 8 & 0.09 & 41 & 0.47 \\
\hline Aegithinidae & $\begin{array}{l}\text { Aegithina } \\
\text { viridissima }\end{array}$ & Green iora & 89 & 1.02 & 17 & 0.19 & 7 & 0.08 \\
\hline Turdidae & Copsychus saularis & $\begin{array}{l}\text { Oriental magpie } \\
\text { robin }\end{array}$ & 84 & 0.96 & 19 & 0.22 & 13 & 0.15 \\
\hline Meropidae & Merops philippinus & $\begin{array}{l}\text { Blue-tailed } \\
\text { bee-eater }\end{array}$ & 68 & 0.80 & 37 & 0.42 & 142 & 1.63 \\
\hline Rhipiduridae & Rhipidura javanica & Pied fantail & 65 & 0.74 & 23 & 0.26 & 8 & 0.09 \\
\hline Cisticolidae & Prinia flaviventris & $\begin{array}{l}\text { Yellow-bellied } \\
\text { prinia }\end{array}$ & 65 & 0.74 & 21 & 0.24 & 20 & 0.23 \\
\hline Laniidae & Lanius cristatus & Brown shrike & 64 & 0.73 & 16 & 0.18 & 12 & 0.14 \\
\hline Rallidae & Gallinula chloropus & $\begin{array}{l}\text { Common } \\
\text { moorhen }\end{array}$ & 61 & 0.70 & 28 & 0.32 & 6 & 0.07 \\
\hline Oriolidae & Oriolus chinensis & $\begin{array}{l}\text { Black-napped } \\
\text { oriole }\end{array}$ & 59 & 0.68 & 11 & 0.13 & 14 & 0.16 \\
\hline Cuculidae & $\begin{array}{l}\text { Centropus } \\
\text { bengalensis }\end{array}$ & Lesser coucal & 48 & 0.55 & 0 & 0 & 12 & 0.14 \\
\hline Sturnidae & $\begin{array}{l}\text { Acridotheres } \\
\text { grandis }\end{array}$ & $\begin{array}{l}\text { White-vented } \\
\text { myna }\end{array}$ & 44 & 0.50 & 7 & 0.08 & 6 & 0.07 \\
\hline Anatidae & $\begin{array}{l}\text { Dendrocygna } \\
\text { javanica }\end{array}$ & $\begin{array}{l}\text { Lesser whistling } \\
\text { duck }\end{array}$ & 37 & 0.42 & 0 & 0 & 199 & 2.28 \\
\hline
\end{tabular}


TABle 1: Continued.

\begin{tabular}{|c|c|c|c|c|c|c|c|c|}
\hline \multirow{3}{*}{ Family name } & \multirow{3}{*}{ Scientific name } & \multirow{3}{*}{ Common name } & \multicolumn{6}{|c|}{ No. of observations with habitat } \\
\hline & & & \multicolumn{2}{|c|}{ Marsh swamp } & \multicolumn{2}{|c|}{ Lotus swamp } & \multicolumn{2}{|c|}{ Open water body } \\
\hline & & & Observation & $\begin{array}{c}\% \text { of all } \\
\text { detection }\end{array}$ & Observation & $\begin{array}{c}\% \text { of all } \\
\text { detection }\end{array}$ & Observation & $\begin{array}{l}\% \text { of all } \\
\text { detection }\end{array}$ \\
\hline Ardeidae & $\begin{array}{l}\text { Ixobrychus } \\
\text { cinnamomeus }\end{array}$ & $\begin{array}{l}\text { Cinnamon } \\
\text { bittern }\end{array}$ & 28 & 0.32 & 0 & 0 & 6 & 0.07 \\
\hline Aegithinidae & Aegithina tiphia & Common iora & 28 & 0.32 & 3 & 0.03 & 5 & 0.06 \\
\hline Picidae & $\begin{array}{l}\text { Dinopium } \\
\text { javanense }\end{array}$ & $\begin{array}{l}\text { Common } \\
\text { flameback }\end{array}$ & 27 & 0.31 & 10 & 0.11 & 3 & 0.03 \\
\hline Sylviidae & $\begin{array}{l}\text { Acrocephalus } \\
\text { orientalis }\end{array}$ & $\begin{array}{l}\text { Oriental reed } \\
\text { warbler }\end{array}$ & 24 & 0.27 & 1 & 0 & 1 & 0.01 \\
\hline Rallidae & Porzana cinerea & $\begin{array}{l}\text { White-browed } \\
\text { crake }\end{array}$ & 24 & 0.27 & 5 & 0.07 & 0 & 0 \\
\hline Phasianidae & Gallus gallus & Red jungle-fowl & 21 & 0.24 & 8 & 0.09 & 7 & 0.08 \\
\hline Ardeidae & Butorides striatus & Little heron & 20 & 0.23 & 3 & 0.03 & 0 & 0 \\
\hline Campephagidae & e Lalage nigra & Pied triller & 19 & 0.22 & 0 & 0 & 7 & 0.08 \\
\hline Columbidae & Treron bicincta & $\begin{array}{l}\text { Orange- } \\
\text { breasted green } \\
\text { pigeon }\end{array}$ & 17 & 0.19 & 15 & 0.17 & 0 & 0 \\
\hline Sylviidae & $\begin{array}{l}\text { Orthotomus } \\
\text { ruficeps }\end{array}$ & Ashy tailorbird & 14 & 0.16 & 4 & 0.05 & 0 & 0 \\
\hline Ardeidae & $\begin{array}{l}\text { Nycticorax } \\
\text { nycticorax }\end{array}$ & $\begin{array}{l}\text { Black-crowned } \\
\text { nightheron }\end{array}$ & 13 & 0.15 & 0 & 0 & 0 & 0 \\
\hline Nectariniidae & Nectarinia jugularis & $\begin{array}{l}\text { Olive-backed } \\
\text { sunbird }\end{array}$ & 13 & 0.15 & 1 & 0.01 & 2 & 0.02 \\
\hline Cuculidae & $\begin{array}{l}\text { Cacomantis } \\
\text { merulinus }\end{array}$ & Plaintive cuckoo & 12 & 0.14 & 13 & 0.15 & 0 & 0 \\
\hline Anatidae & $\begin{array}{l}\text { Nettapus } \\
\text { coromandelianus }\end{array}$ & $\begin{array}{l}\text { Cotton pygmy } \\
\text { goose }\end{array}$ & 11 & 0.13 & 1 & 0.01 & 81 & 0.93 \\
\hline Meropidae & Merops viridis & $\begin{array}{l}\text { Blue-throated } \\
\text { bee-eater }\end{array}$ & 10 & 0.10 & 9 & 0.10 & 0 & 0 \\
\hline Scolopacidae & Gallinago stenura & Pintail snipe & 10 & 0.10 & 0 & 0 & 2 & 0.02 \\
\hline Nectariniidae & $\begin{array}{l}\text { Anthreptes } \\
\text { malacensis }\end{array}$ & $\begin{array}{l}\text { Brown-throated } \\
\text { sunbird }\end{array}$ & 8 & 0.09 & 3 & 0.03 & 0 & 0 \\
\hline Corvidae & $\begin{array}{l}\text { Corvus } \\
\text { macrorhynchos }\end{array}$ & $\begin{array}{l}\text { Large-billed } \\
\text { crow }\end{array}$ & 8 & 0.09 & 0 & 0 & 12 & 0 \\
\hline Columbidae & Treron olax & $\begin{array}{l}\text { Little green } \\
\text { pigeon }\end{array}$ & 8 & 0.09 & 1 & 0.01 & 0 & 0 \\
\hline Estrildidae & Lonchura maja & $\begin{array}{l}\text { White-headed } \\
\text { munia }\end{array}$ & 8 & 0.09 & 0 & 0 & 0 & 0 \\
\hline Turnicidae & Turnix suscitator & $\begin{array}{l}\text { Barred button } \\
\text { quail }\end{array}$ & 7 & 0.08 & 1 & 0.01 & 0 & 0 \\
\hline Ardeidae & Ardea cinerea & Grey heron & 7 & 0.08 & 4 & 0.05 & 1 & 0.01 \\
\hline Sylviidae & $\begin{array}{l}\text { Orthotomus } \\
\text { sutorius }\end{array}$ & $\begin{array}{l}\text { Common } \\
\text { tailorbird }\end{array}$ & 6 & 0.07 & 2 & 0.02 & 3 & 0.03 \\
\hline Pycnonotidae & $\begin{array}{l}\text { Pycnonotus } \\
\text { plumosus }\end{array}$ & $\begin{array}{l}\text { Olive-winged } \\
\text { bulbul }\end{array}$ & 6 & 0.07 & 0 & 0 & 0 & 0 \\
\hline Sylviidae & $\begin{array}{l}\text { Orthotomus } \\
\text { sericeus }\end{array}$ & $\begin{array}{l}\text { Rufous-tailed } \\
\text { tailorbird }\end{array}$ & 6 & 0.07 & 0 & 0 & 0 & 0 \\
\hline Ardeidae & $\begin{array}{l}\text { Ixobrychus } \\
\text { eurhythmus }\end{array}$ & $\begin{array}{l}\text { Schrenck's } \\
\text { bittern }\end{array}$ & 6 & 0.07 & 0 & 0 & 1 & 0.01 \\
\hline Cuculidae & $\begin{array}{l}\text { Chrysococcyx } \\
\text { minutillus }\end{array}$ & $\begin{array}{l}\text { Little bronze } \\
\text { cuckoo }\end{array}$ & 5 & 0.06 & 2 & 0.02 & 2 & 0.02 \\
\hline Cisticolidae & Cisticola juncidis & Zitting cisticola & 5 & 0.06 & 0 & 0 & 5 & 0.06 \\
\hline Accipitridae & Elanus caeruleus & $\begin{array}{l}\text { Black-shoulder } \\
\text { kite }\end{array}$ & 4 & 0.05 & 0 & 0 & 0 & 0 \\
\hline
\end{tabular}


Table 1: Continued.

\begin{tabular}{|c|c|c|c|c|c|c|c|c|}
\hline \multirow{3}{*}{ Family name } & \multirow{3}{*}{ Scientific name } & \multirow{3}{*}{ Common name } & \multicolumn{6}{|c|}{ No. of observations with habitat } \\
\hline & & & \multicolumn{2}{|c|}{ Marsh swamp } & \multicolumn{2}{|c|}{ Lotus swamp } & \multicolumn{2}{|c|}{ Open water body } \\
\hline & & & Observation & $\begin{array}{c}\% \text { of all } \\
\text { detection }\end{array}$ & Observation & $\begin{array}{l}\% \text { of all } \\
\text { detection }\end{array}$ & Observation & $\begin{array}{l}\% \text { of all } \\
\text { detection }\end{array}$ \\
\hline Coraciidae & $\begin{array}{l}\text { Eurystomus } \\
\text { orientalis }\end{array}$ & Dollar bird & 4 & 0.05 & 0 & 0 & 0 & 0 \\
\hline Ardeidae & Egretta alba & Great egret & 4 & 0.05 & 0 & 0 & 0 & 0 \\
\hline Cuculidae & Centropus sinensis & Greater coucal & 4 & 0.05 & 0 & 0 & 2 & 0.02 \\
\hline Ardeidae & Egretta garzetta & Little egret & 4 & 0.05 & 0 & 0 & 0 & 0 \\
\hline Pachycephalidae & $\begin{array}{l}\text { Pachycephala } \\
\text { grisola }\end{array}$ & $\begin{array}{l}\text { Mangrove } \\
\text { whistler }\end{array}$ & 4 & 0.05 & 0 & 0 & 1 & 0.01 \\
\hline Nectariniidae & Anthreptes simplex & Plain sunbird & 4 & 0.05 & 0 & 0 & 0 & 0 \\
\hline Rallidae & Gallicrex cinerea & Water cock & 4 & 0.05 & 1 & 0.01 & 3 & 0.03 \\
\hline Rallidae & Porzana pusilla & Baillon's crake & 3 & 0.03 & 11 & 0.13 & 0 & 0 \\
\hline Nectariniidae & Aethopyga saturata & $\begin{array}{l}\text { Black-throated } \\
\text { sunbird }\end{array}$ & 3 & 0.03 & 0 & 0 & 0 & 0 \\
\hline Caprimulgidae & Caprimulgus affinis & $\begin{array}{l}\text { Savanna } \\
\text { nightjar }\end{array}$ & 3 & 0.03 & 0 & 0 & 1 & 0.01 \\
\hline Muscicapidae & $\begin{array}{l}\text { Muscicapa } \\
\text { dauurica }\end{array}$ & $\begin{array}{l}\text { Asian brown } \\
\text { flycatcher }\end{array}$ & 2 & 0.02 & 2 & 0.02 & 0 & 0 \\
\hline Alcedinidae & Alcedo atthis & $\begin{array}{l}\text { Common } \\
\text { kingfisher }\end{array}$ & 2 & 0.02 & 1 & 0.01 & 0 & 0 \\
\hline Sturnidae & Gracula religiosa & Hill myna & 2 & 0.02 & 0 & 0 & 0 & 0 \\
\hline Caprimulgidae & $\begin{array}{l}\text { Caprimulgus } \\
\text { macrurus }\end{array}$ & $\begin{array}{l}\text { Large-tailed } \\
\text { nightjar }\end{array}$ & 2 & 0.02 & 0 & 0 & 1 & 0.01 \\
\hline Podicipedidae & $\begin{array}{l}\text { Tachybaptus } \\
\text { ruficollis }\end{array}$ & Little grebe & 2 & 0.02 & 2 & 0.02 & 7 & 0.08 \\
\hline Laniidae & Lanius schach & $\begin{array}{l}\text { Long-tailed } \\
\text { shrike }\end{array}$ & 2 & 0.02 & 1 & 0.01 & 0 & 0 \\
\hline Dicruridae & $\begin{array}{l}\text { Dicrurus } \\
\text { leucophaeus }\end{array}$ & Ashy drongo & 1 & 0.01 & 0 & 0 & 0 & 0 \\
\hline Accipitridae & Aviceda leuphotes & Black baza & 1 & 0.01 & 0 & 0 & 1 & 0.01 \\
\hline Phasianidae & Coturnix chinensis & $\begin{array}{l}\text { Blue-breasted } \\
\text { quail }\end{array}$ & 1 & 0.01 & 0 & 0 & 2 & 0.02 \\
\hline Corvidae & Corvus splendens & House crow & 1 & 0.01 & 0 & 0 & 3 & 0.03 \\
\hline Sylviidae & $\begin{array}{l}\text { Phylloscopus } \\
\text { inornatus }\end{array}$ & $\begin{array}{l}\text { Inornate } \\
\text { warbler }\end{array}$ & 1 & 0.01 & 0 & 0 & 0 & 0 \\
\hline Nectariniidae & $\begin{array}{l}\text { Arachnothera } \\
\text { longirostra }\end{array}$ & $\begin{array}{l}\text { Little } \\
\text { spiderhunter }\end{array}$ & 1 & 0.01 & 1 & 0.01 & 0 & 0 \\
\hline Jacanidae & $\begin{array}{l}\text { Hydrophasianus } \\
\text { chirurgus }\end{array}$ & $\begin{array}{l}\text { Pheasant-tailed } \\
\text { jacana }\end{array}$ & 1 & 0.01 & 6 & 0.07 & 0 & 0 \\
\hline Sylviidae & Locustella certhiola & $\begin{array}{l}\text { Rusty-rumped } \\
\text { warbler }\end{array}$ & 1 & 0.01 & 1 & 0.01 & 0 & 0 \\
\hline Picidae & $\begin{array}{l}\text { Picumnus } \\
\text { innominatus }\end{array}$ & Speckled piculet & 1 & 0.01 & 0 & 0 & 0 & 0 \\
\hline Scolopacidae & Tringa. hypoleucos & $\begin{array}{l}\text { Common } \\
\text { sandpiper }\end{array}$ & 0 & 0.01 & 2 & 0.02 & 0 & 0 \\
\hline Picidae & Celeus brachyurus & $\begin{array}{l}\text { Rufous } \\
\text { woodpecker }\end{array}$ & 0 & 0.00 & 2 & 0.02 & 0 & 0 \\
\hline Columbidae & Treron curvirostra & $\begin{array}{l}\text { Thick-billed } \\
\text { green Pigeon }\end{array}$ & 0 & 0.00 & 1 & 0.01 & 0 & 0 \\
\hline Nectariniidae & $\begin{array}{l}\text { Nectarinia } \\
\text { calcostetha }\end{array}$ & $\begin{array}{l}\text { Copper- } \\
\text { throated } \\
\text { sunbird }\end{array}$ & 0 & 0.00 & 1 & 0.01 & 0 & 0 \\
\hline Campephagidae & $\begin{array}{l}\text { Pericrocotus } \\
\text { divaricatus }\end{array}$ & Ashy minivet & 0 & 0.00 & 0 & 0 & 3 & 0.03 \\
\hline & Total & & 6086 & & 1097 & & 1545 & \\
\hline
\end{tabular}


TABLE 2: List of microclimate and microhabitat variables of marsh swamp, lotus swamp, and open water body at the Paya Indah Wetland Reserve, Peninsular Malaysia.

\begin{tabular}{|c|c|c|c|c|}
\hline \multirow[t]{2}{*}{ S. no } & \multirow[t]{2}{*}{ Variables } & \multicolumn{3}{|c|}{ Mean value (range) } \\
\hline & & Marsh swamp & Lotus swamp & Open water body \\
\hline \multirow[t]{4}{*}{1} & Microclimate variables & & & \\
\hline & (a) Mean temperature $\left({ }^{\circ} \mathrm{C}\right)$ & $28.1^{\circ} \mathrm{C}\left(25-31^{\circ} \mathrm{C}\right)$ & $27.50^{\circ} \mathrm{C}\left(26-30^{\circ} \mathrm{C}\right)$ & $28.5^{\circ} \mathrm{C}\left(26.5-30^{\circ} \mathrm{C}\right)$ \\
\hline & (b) Mean relative humidity (\%) & $95.3 \%(89-97 \%)$ & $96.45 \%(94-97 \%)$ & $95.5 \%(94-97 \%)$ \\
\hline & (c) Mean light intensity (LUX) & 233.65 (16-520 Lux) & 260.60 (150-362 Lux $)$ & $438.16(351-517$ Lux $)$ \\
\hline \multirow[t]{15}{*}{2} & Microhabitat variables & & & \\
\hline & (A) Vegetation cover percentage & $81.3 \%$ & $77.0 \%$ & $76.7 \%$ \\
\hline & (a) Emergent vegetation (EMR) & $59.1 \%$ & $55.4 \%$ & $30.8 \%$ \\
\hline & (b) Submerged vegetation (SUB) & $13.6 \%$ & $15.4 \%$ & $61.5 \%$ \\
\hline & (c) Grasses (GRS) & $4.8 \%$ & $5.0 \%$ & $4.5 \%$ \\
\hline & (d) Shrubs (SHU) & $3.7 \%$ & $1.2 \%$ & $3.2 \%$ \\
\hline & (B) Number of trees & 67 & 17 & 6 \\
\hline & (i) Diameter $(\mathrm{cm})$ & & & \\
\hline & (a) Diameter $(<15 \mathrm{~cm})(\mathrm{DA})$ & 51 & 0 & 5 \\
\hline & (b) Diameter $(16-30 \mathrm{~cm})(\mathrm{DB})$ & 12 & 15 & 1 \\
\hline & (c) Diameter $(31-45 \mathrm{~cm})(\mathrm{DC})$ & 4 & 2 & 0 \\
\hline & (ii) Height (meter) & & & \\
\hline & (a) Height $(<10 \mathrm{~m})$ (HA) & 50 & 13 & 3 \\
\hline & (b) Height (11-20 m) (HB) & 16 & 4 & 1 \\
\hline & (c) Height $(21-30 \mathrm{~m})(\mathrm{HC})$ & 1 & 0 & 2 \\
\hline
\end{tabular}

with the emergent vegetation, while oriental reed warblers, baya weavers, grey herons, yellow-bellied prinias and cinnamon bitterns showed a close association with the emergent vegetation and trees. Furthermore, rufous-tailed tailorbirds, plaintive cuckoos, blue-throated bee-eaters, lesser coucals, common flamebacks, brown-throated sunbirds and orangebreasted green pigeons showed a close relationship with the humid areas dominated by trees having the diameter of 16$30 \mathrm{~cm}$ and the height of $<10 \mathrm{~m}$. Oriental magpie robins, white-breasted waterhens, Richard's pipits, red junglefowl, barred button quails, yellow-vented bulbuls and spotted doves indicated strong association with the grassy areas. Moreover, pink-necked green pigeons, jungle mynas, blacknaped orioles, orange-breasted Green Pigeons and Whitevented Mynas showed a strong relationship with the shrubs. Pintail Snipes, common ioras, pied trillers, common tailorbirds and pied fantails also showed a close relationship with light intensity, while blue-tailed bee-eaters, white-throated kingfishers, philippine glossy starlings, ashy tailorbirds, and green ioras indicated an association with the densely vegetated areas (Figure 2).

3.8. Correlation of Birds, Microclimate, and Microhabitat in Lotus Swamp. The ordination biplot diagram of lotus swamp habitat showed that red-wattled lapwings and white-vented mynas had high relationship with temperature. Notably, blue-tailed bee-eaters, orange-breasted green pigeons, pacific swallows, plaintive cuckoos, yellow-bellied prinias and pinknecked green pigeons showed a strong association with the trees (having height $11-20 \mathrm{~m}$ ), shrubs and also light intensity. In addition, common flamebacks, black-naped orioles, spotted doves and brown shrikes also showed a positive relationship with the shrubs and trees. Blue-throated beeeaters, oriental magpie robins, eurasian tree sparrows, whitethroated kingfishers and peaceful doves indicated a significant correlationship with the trees having the diameter $<15 \mathrm{~cm}$ and the height $<10 \mathrm{~m}$. Pied fantails and yellowvented bulbuls also had a strong relationship with relative humidity and vegetation cover greater than $75 \%$. Additionally, white-breasted waterhens, white-browed crakes, purple swamphens and pheasant-tailed jacanas were highly correlated with the emergent vegetation. Red junglefowl, baya weavers, scaly-breasted munias, grey herons, and jungle mynas had a close relationship with the area dominated by grasses (Figure 3).

3.9. Correlationship of Birds, Microclimate, and Microhabitat in Open Water Body. The RDA biplot diagram of the open water body habitat revealed that purple swamphens, purple herons, yellow bitterns, cinnamon bitterns and yellow-bellied prinias had a strong association with the emergent vegetated areas having cover of $25-50 \%$. In addition, common moorhens and yellow-bellied prinias had an association with the emergent vegetation and grasses. Meanwhile, scalybreasted munias, peaceful doves, oriental magpie robins, spotted doves, Richard's pipits, lesser coucals, brown shrikes and common mynas were also highly related with the grasses. Cotton pygmy geese, little grebes and lesser whistling ducks indicated a close relationship with the submerged vegetation, light intensity and temperature. On the other hand, pied fantails, pink-necked green pigeons and yellow-vented bulbuls highlighted a strong association with the shrubs. Baya 


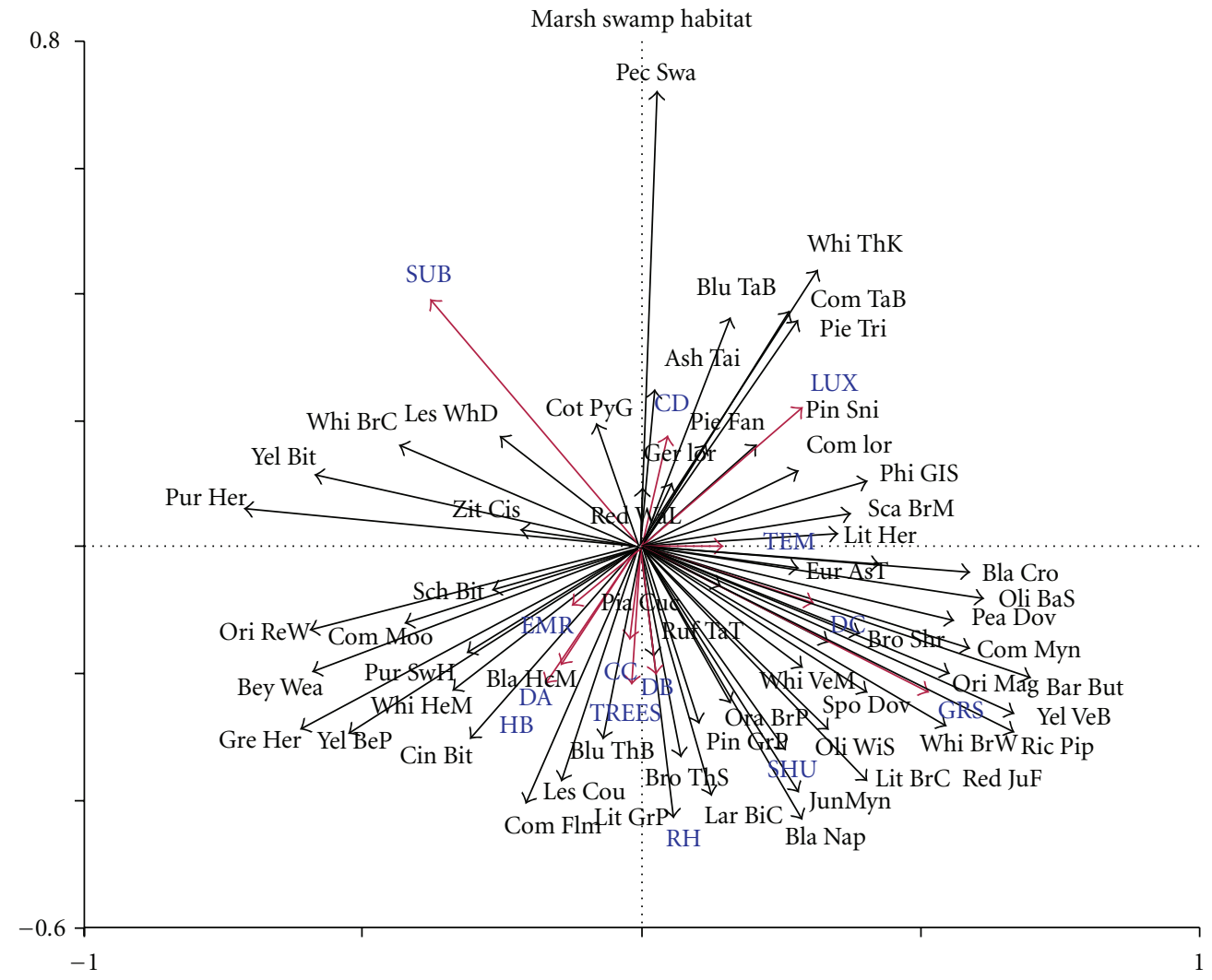

Figure 2: Ordination plot from a redundancy analysis of the distribution of birds of marsh swamp in relation to 14 environmental variables (for details see Table 2). The biplot of axes 1 and 2 is presented; the orientation of each variable in relation to each of these axes is presented by an arrow, the length of which indicates the degree of correlation with those axes.

weavers, blue-tailed Bee-eaters, pied trillers, red junglefowl and green ioras showed a close link with the trees (having diameter of $16-30 \mathrm{~cm}$ and the height of $11-20 \mathrm{~m}$ ), and the vegetation cover of 51-75\%. Jungle mynas, large-billed crows, white-vented mynas, Philippine glossy starlings, and black-naped orioles indicated a strong association with the humid area dominated by trees having the diameter of $<15 \mathrm{~cm}$ and the height of $<10 \mathrm{~m}$ (Figure 4 ).

\section{Discussions}

It is necessary to integrate spatial distribution with species characteristics in the analysis of factors responsible for distribution of bird species in wetlands. About $40 \%$ of the lakes at the Paya Indah Wetland Reserve are open water bodies, dominated by submerged and floating vegetation such as pond weeds (Potamogeton sp.), water milfoils (Myriophyllum sp.), bladderworts (Utricularia sp.), rushes (Scirpus sp), coontails (Ceratophyllum sp.), and waterweeds or hydrillas (Hydrilla sp.). Water level changed from time to time mainly depending on the rainfall pattern. The ratio between the emergent vegetation and open water $(40: 60)$ was also the key factor that influenced the distribution of waterbirds in the wetland area. About $60 \%$ of the water bodies were densely covered by emergent vegetation, such as water chestnuts, marsh sedges, water lilies, water-milfoils, bulrushes and phragmites. The emergent vegetation was an important habitat for the swamphens, moorhens, crakes, herons, and bitterns. These waterbird species used the emergent vegetation for different purposes, such as hunting, perching, and escape cover.

Previously, several studies have demonstrated an association between bird species distribution and wetland habitats $[40,41]$. Recently, attention has been given on habitat interaction especially to examine the difference in habitat use among the coexisting species [42-44]. In this study, the results obtained using the canonical correspondence analysis highlighted that the plant species (e.g., emergent vegetation, submerged vegetation, grasses, shrubs, and trees composition), vegetation structures (e.g., diameter and height), and climatic variables (e.g., temperature, relative humidity, and light intensity) were the main factors that influenced the distribution of waterbirds and terrestrial birds in the wetland reserve. The results also indicated that each species had adapted a fairly unique set of microhabitat and microclimate conditions. For instance, the lesser whistling ducks used the water body habitat dominated by submerged vegetation for foraging and loafing on dead trees and reed beds of the emergent vegetation along the edges. They preferred to forage in the morning, and, when the temperature rose, their activity turned to loafing. Similarly, the purple swamphens 


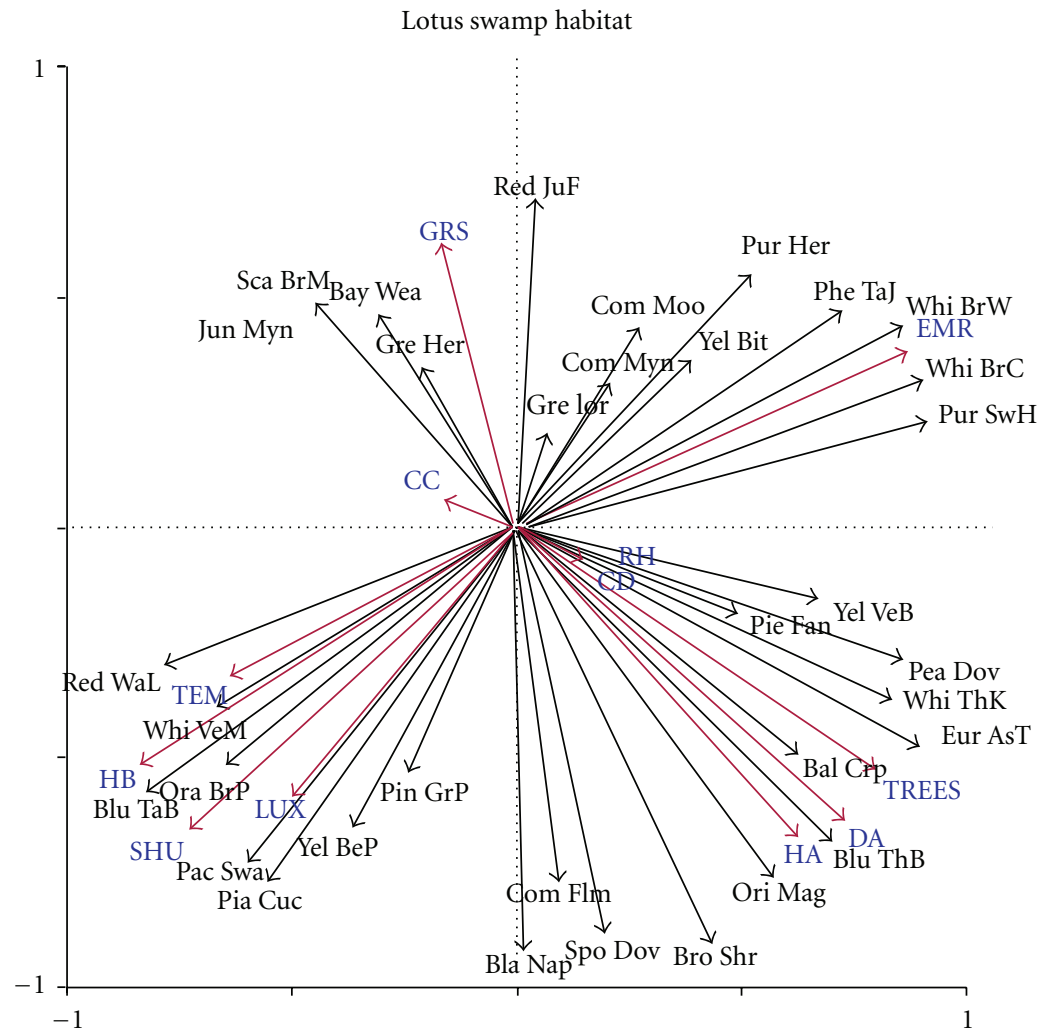

FIGURE 3: Ordination plot from a redundancy analysis of the distribution of birds of lotus swamp in relation to 14 environmental variables (for details see Table 2). The biplot of axes 1 and 2 is presented; the orientation of each variable in relation to each of these axes is presented by an arrow, the length of which indicates the degree of correlation with those axes.

preferred to use the marsh swamp habitat dominated by emergent vegetation. This revealed that the microhabitat and microclimate selection might vary from species to species.

The results further showed that the waterbirds, for instance, the ducks, geese, and grebes, had a strong correlationship with the submerged vegetation, temperature, and light intensity. This might be due to greater abundance and higher diversity of macroinvertebrates and fish. The macroinvertebrates of the Paya Indah Wetland Reserve consisted of snails, arachnids, insect larvae, and crustaceans. The higher abundance and richness of macro-invertebrates and fish occurred in the submerged vegetation [45-47]. Apparently, relative abundance of ducks and geese was strongly related to the domination of submerged vegetation in open water bodies, and they preferred to forage in submerged vegetation for food items [48-51]. The submerged vegetation supported the complex trophic structure in the wetland [52], and it was an important source of food for invertebrates such as insects, isopods, decapods, crustaceans, and molluscs, as well as fish and birds [53-55].

Anatidae avoided the dense emergent vegetation and preferred submerged vegetation instead because this vegetative cover potentially restricted the movement and foraging efficiency of the birds in the study area. Such types of finding also have been reported by Van Rees-Siewert and Dinsmore [56], King and Wrubleski [57] and Benoit and Askins [58]. Dense vegetation might interfere with the movement and foraging efficiency of the waterbirds. The swamphens, bitterns, moorhens, jacanas, herons, crakes, warblers, prinias and munias showed a positive association with the emergent vegetation in the wetland area. This might be due to diverse food resources occuring in emergent vegetation (e.g., amphibians, fish, and aquatic invertebrates, such as snails, insects larvae, crustaceans, and aquatic annelids), refuge from predators and potential nursery sites for their chicks. The vegetation with moderate to low structure offered suitable foraging opportunities particularly for the herons, bitterns, swamphens, and crakes in the study area. This might also be due assigned to the richness and abundance of aquatic invertebrates, amphibians, and fish in the emergent vegetation.

Additionally, the swamphens, moorhens, and crakes were observed using the dense stands of emergent vegetation $[59,60]$. This was because the dense emergent vegetation provided a hiding cover from predator's visual detection [61]. Apparently, the herons, bitterns, and egrets also selected the emergent vegetation with shallow water for their foraging activity [62]. Needless to say, herons and egrets have long bills and stalks that enable them to submerge for prey while wading in shallow water [63] and capturing their prey by doing a direct head movement $[64,65]$.

The results showed that the terrestrial birds, such as the minivets, tailorbirds, kites, bee-eaters, and crows, were strongly associated with the trees in the study area. This 


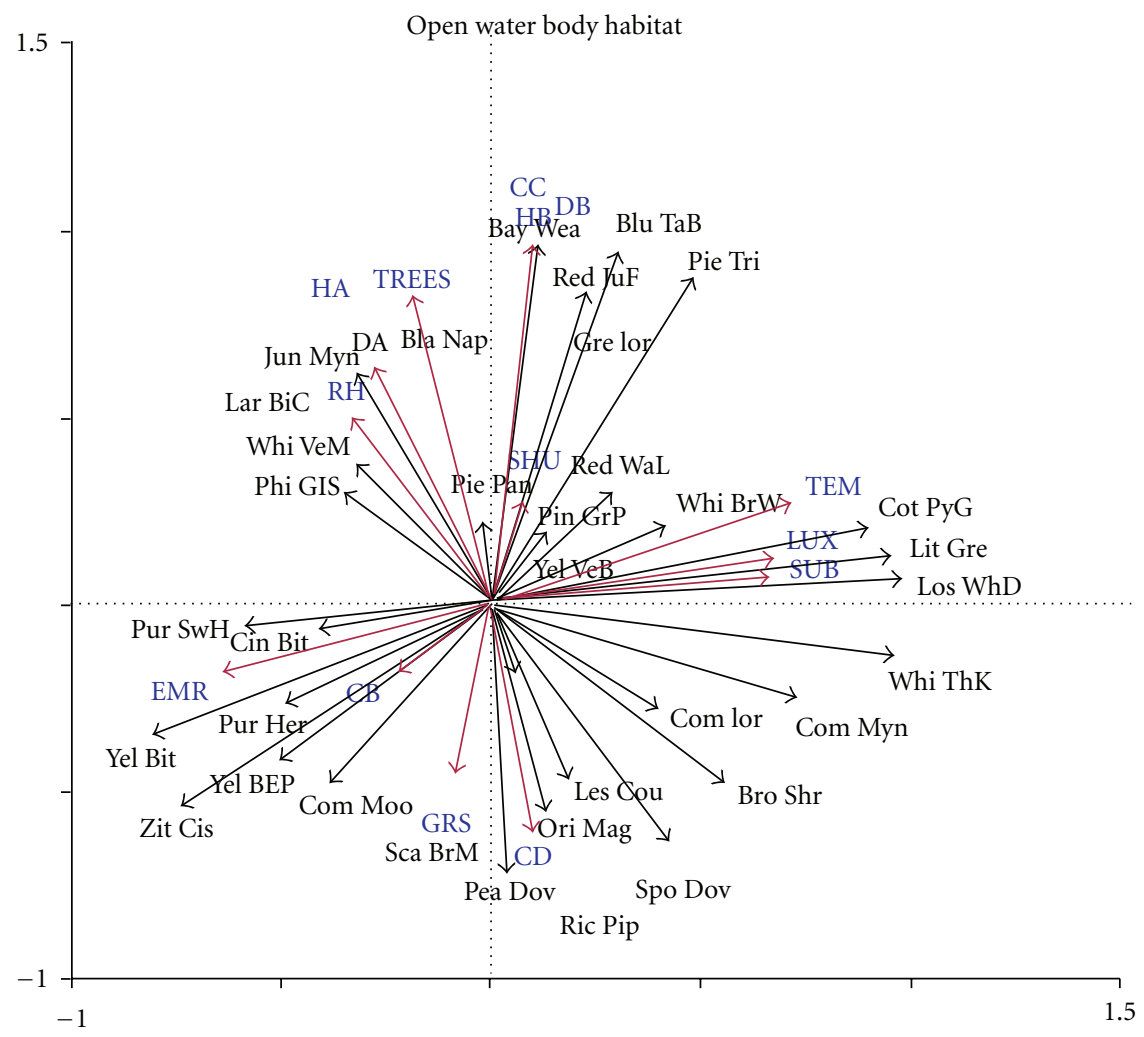

Figure 4: Ordination plot from a redundancy analysis of the distribution of birds of open water body in relation to 14 environmental variables (for details see Table 2). The biplot of axes 1 and 2 is presented; the orientation of each variable in relation to each of these axes is presented by an arrow, the length of which indicates the degree of correlation with those axes.

might be due to the trees that attracted diverse insects and provided suitable foraging surfaces, shelters, and nesting sites for the bird species. Moreover, the weavers, munias, cuckoos, coucals, lapwings, snipes, and waterhens showed a positive correlationship with the emergent vegetation, grasses, relative humidity, temperature, and ground vegetation cover. The grasses provided a variety of flowers and seeds that attracted the insects, whereby the seeds and insects were the main sources of energy for these bird species.

The bee-eaters, fantails, dollar birds, mynas, sparrows, kingfishers, orioles, pigeons, starlings, bulbuls, and trillers indicated a strong relationship with the shrubs, trees, relative humidity, light intensity, and ground vegetation cover in the wetland reserve. The reason was that these birds preferred different microhabitats like the marsh swamp, lotus swamp, open water body, dryland and patches of shrubs, and microclimate, such as the temperature, humidity, and light intensity. The shrubs and trees provided a diversity of flowers and fruits that attracted a wide array of insects. The berries and insects were the main food resources for these birds. In addition, the shrubs and trees provided hiding covers from predators and inclement weather and suitable nesting sites for them.

The robins, doves, junglefowl, coucals, pipits, munias, shrikes, quails, and mynas indicated a strong association with the grasses, shrubs, trees, relative humidity, light intensity, and ground vegetation. The variety of vegetation [66], vertical zonation of shrubs and tree vegetation [67], structural characteristics of vegetation such as diameter and height, distribution of trees [68], food, and nutrients [69], and amount of ground cover [70] were identified as important factors that directly and indirectly influenced the occurrence and distribution of the birds in the wetland reserve.

The vegetation structure and floristic composition were the key factors that affected the habitat selection of the birds and indicated where and how the birds used the resources $[71,72]$. Microclimatic factors such as the temperature, rainfall, relative humidity, and microhabitat factors, for instance, vegetation cover, had played important roles in the distribution of prey and bird species in the wetland ecosystem $[73,74]$. The light intensity and temperature affects on foraging behaviour, species dispersal and habitat selection, reproduction, and timing of breeding season of avian species. Birds may respond directly to microclimate changes or indirectly to changes in food or cover resulting from microclimate changes [75]. Karr and Freemark [76] suggested the importance of microclimate as a factor in determining avian "physiological comfort." Extremes in microclimate may have adverse effects on birds and their reproductive fitness [7779]. Feeding in open areas where the incoming solar radiation and air temperature are greater than the adjacent vegetated area [80] energetically reduces foraging costs [81]. In addition, other factors such as the weather (rainfall), social interactions $[82,83]$, and predators also played important 
TABle 3: List of vegetation recorded at the Paya Indah Wetland Reserve, Peninsular Malaysia.

\begin{tabular}{|c|c|c|}
\hline Family name & Scientific name & Local name \\
\hline \multicolumn{3}{|c|}{ Tree Species } \\
\hline Anacardiaceae & Mangifera indica & Mangga \\
\hline Annonaceae & Cananga odorata & Kenanga \\
\hline \multirow[t]{2}{*}{ Apocynaceae } & Alstonia scholaris & Pohon \\
\hline & Dyera costulata & Jelutong \\
\hline Araliaceae & Schefflera heterophylla & \\
\hline \multirow[t]{2}{*}{ Arecaceae } & Cocos nucifera & Coconut tree \\
\hline & Elaeis guineensis & Oil Palm \\
\hline Casuarinaceae & Casuarina equisetifolia & Rhu \\
\hline \multirow[t]{3}{*}{ Clusiaceae } & Cratoxylum maingayi & Geronggang \\
\hline & Mesua elegans & Penaga \\
\hline & Clusia rosea & Pitch apple \\
\hline Combretaceae & Terminalia muelleri & Jelawai \\
\hline \multirow[t]{3}{*}{ Dipterocarpaceae } & Shorea materialis & Meranti temak \\
\hline & Shorea sumatrana & Balav sengkawang air \\
\hline & Shorea ochrohloia & Seraya Batu \\
\hline Ebenaceae & Diospyros argentea & Bedil lalat \\
\hline \multirow[t]{2}{*}{ Elaeocarpaceae } & Elaecocarpus floribundus & Mendung \\
\hline & Elaeocarpus nitidus & Mendung \\
\hline \multirow[t]{3}{*}{ Euphorbiaceae } & Macaranga tanarius & Blush \\
\hline & Mallotus paniculatus & Balik angin \\
\hline & Pimelodendron griffithianum & Perahikan \\
\hline Fabaceae & Delonix regia & Semarak api \\
\hline Lauraceae & Cinnamomum iners & Medang teja \\
\hline Lecythidaceae & Barringtonia edulis & Putat \\
\hline \multirow[t]{10}{*}{ Leguminosae } & Acacia auriculiformis & Acacia \\
\hline & Cassia javanica & Pink shower \\
\hline & Cassia fistula & Amaltas \\
\hline & Acacia mangium & Acacia \\
\hline & Albizia julibrissin & Batai \\
\hline & Saraca cauliflora & Gapis \\
\hline & Sindora wallichii & Spetir daun tebal \\
\hline & Cynometra malaccensis & Kekatong \\
\hline & Tamarindus indica & Asam jawa \\
\hline & Flemingia macrophylla & Serengan jantan \\
\hline Loganiaceae & Fragraea fragrans & Tembusu \\
\hline Lythraceae & Lagerstroemia speciosa & Bungor \\
\hline Malvaceae & Sterculia foetida & Kelumpang jari \\
\hline Meliaceae & Azadirachta excelsa & Sentang \\
\hline \multirow[t]{7}{*}{ Moraceae } & Artocarpus maingayi & Pudu \\
\hline & Ficus benjamina & Ara \\
\hline & Ficus fistulosa & Ara \\
\hline & Streblus elongatus & Tempinis \\
\hline & Artocarpus lanceifolius & Keledang \\
\hline & Ficus maclellandii & Ara \\
\hline & Ficus rubiginosa & Ara \\
\hline
\end{tabular}


Table 3: Continued.

\begin{tabular}{|c|c|c|}
\hline Family name & Scientific name & Local name \\
\hline \multirow[t]{9}{*}{ Myrtaceae } & Syzygium linocieroides & Kelat \\
\hline & Syzygium campanulatum & Kelat \\
\hline & Syzygium hanfii & Kelat \\
\hline & Syzygium lineatum & Kelat \\
\hline & Melaleuca cajuputi & Kayu putih (gelam) \\
\hline & Syzygium grande & Kelat jambu laut \\
\hline & Syzygium polyanthum & Kelat \\
\hline & Syzygium jambos & Kelat jambu \\
\hline & Syzygium microcalyx & Kelat \\
\hline Rutaceae & Melicope glabra & Pepauh \\
\hline \multirow[t]{3}{*}{ Sapindaceae } & Filicium decipiens & Japanese fern leaf tree \\
\hline & Mimusops elengi & Bunga tanjung \\
\hline & Payena lucida & Nyatoh \\
\hline Simaroubaceae & Ailanthus excelsa & Marakuh \\
\hline \multirow[t]{5}{*}{ Sterculiaceae } & Pteroygota alata & Kasah \\
\hline & Streculia lanceolata & Kalumpang \\
\hline & Heritiera javanica & Mengkulang jari \\
\hline & Firmiana malayana & Mata lembu \\
\hline & Sterculia macrophylla & Kelompang \\
\hline \multirow[t]{2}{*}{ Thymelaeaceae } & Gonystylus banacanus & Ramin melawis \\
\hline & Shrub species & \\
\hline Melastomataceae & Melastoma malabathrcium & \\
\hline \multirow[t]{2}{*}{ Dilleniaceae } & Dillenia suffruticosa & \\
\hline & Emergent vegetation & \\
\hline Cyperaceae & Eleocharis dulcis & Water chestnut \\
\hline \multirow[t]{3}{*}{ Nymphaeaceae } & Nelumbo nucifera & Indian or bean or lotus \\
\hline & Nelumbo pubescens & Water lily \\
\hline & Nymphaea rubra & Rubra water lily \\
\hline \multirow[t]{2}{*}{ Philydraceae } & Philydrum lanuginosum & Wooly water lily \\
\hline & Grass Species & \\
\hline Cyperaceae & Scirpus olneyi & Three square bulrush \\
\hline \multirow[t]{2}{*}{ Gramineae } & Imperata cylindrica & Cogon grass \\
\hline & Distichlis spicata & Spike grass \\
\hline \multirow{2}{*}{ Poaceae } & Cynodon dactylon & Carpet grass \\
\hline & Panicum maximum & Buffalo grass \\
\hline 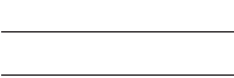 & Sedge Species & \\
\hline Cyperaceae & Scleria purpurascens & Marsh sedge/nut rush \\
\hline \multirow[t]{2}{*}{ Poaceae } & Spartina alterniflora & Rush \\
\hline & Fern and Moss Species & \\
\hline Blechnaceae & Stenochlaena palustris & Climbing fern \\
\hline Thelypteridaceae & Cyclosorus interruptus & Fern \\
\hline Lycopodiaceae & Lycopodium cernuum & Creeping club moss \\
\hline Salviniaceae & Salvinia molesta & Giant or kariba weed \\
\hline \multirow[t]{2}{*}{ Gleicheniaceae. } & Gleichenia linearis & Fern tree \\
\hline & Reed Species & \\
\hline Philydraceae & Phragmites karka & Phragmites \\
\hline Typhaceae & Typha angustifolia & Cattail \\
\hline
\end{tabular}


roles in the distribution and habitat use of the wetland birds in the study area $[28,84,85]$.

Overall, the results of the constrained redundancy ordination analysis indicated that the relationship between the birds and habitats was shaped by microhabitat factors such as ground cover (i.e., the proportion of soil covered with vegetation), plant species richness (i.e., the number of plant species), vegetation type (i.e., trees, shrubs, grasses, emerged and submerged vegetation, sedges, reeds, ferns, and herbs), vegetation structure (i.e., vegetation height and diameter), and microclimate factors (i.e., temperature, humidity, and light intensity). These factors influenced on the distribution, diversity and density, of avian species in the study area.

Besides, other factors, for instance, water quality, such as the water of the some part of lakes was too blackish colour; no bird was observed to use that area. In contrast, nearby other lake having clear water with bluish colour many birds were observed to utilize that area and food resources such as fruiting trees, that is, Ficus, Syzygium, Fragraea, Glabra, and Cinnamons. During fruiting season, these trees abundantly preferred by fruit-eating birds such as bulbuls, starlings, pigeons, and orioles. In addition, such purple swamphen fly from edge to deep water to pluck sink fruit of water lily and bring it to dry place to eat and shelters from harsh weather conditions and predators for example, due to emergence of western marsh harrier, and also during raining bittern, moorhens, and swamphen hided into reed beds and pigeons and doves fly away from foraging sites to dense shrubs and trees to get shelter. These sightings showed that water quality, food resources, and shelter played major roles in the distribution of birds in the study area.

\section{Conclusion}

Marsh swamp was heavily used by wetland bird species (69.7\%) as compared to open water body and lotus swamp. Results revealed that wetland bird species have adapted a fairly unique set of microhabitat characteristics such as vegetation composition and vegetation structures, and microclimate variables are key factors that influenced the species abundance, distribution, and diversity.

\section{Appendices}

\section{A.}

See Table 3 .

\section{B.}

(a) Microclimate Variables. Tem: temperature, RH: relative humidity, LUX: light intensity.

(b) Microhabitat Variables. CB: ground vegetation cover 26$50 \%$, CC: ground vegetation cover 51-75\%, CD: ground vegetation cover 75-100\%, SUB: submerged vegetation, EMR: emergent vegetation, GRA: grasses, SHU: shrubs, DA: tree diameter $<15 \mathrm{~cm}$, DB: tree diameter $16-30 \mathrm{~cm}$, DC: tree diameter 31-45 cm, HA: tree height $<10 \mathrm{~m}$, HB: tree height 11-20 m, HC: tree height 21-30 m.

(c) Bird Species. Ash Tai: ashy tailorbird, Bar But: barred button quail, Bay Wea: baya weaver, Bla Cro: black-crowned nightheron, Bla HeM: black-headed munia, Bla Nap: blacknaped oriole, Blu TaB: blue-tailed bee-eater, Blu ThB: bluethroated bee-eater, Brh Kit: brahminy kite, Bro Shr: brown shrike, Bro ThS: brown-throated sunbird, Che WiC: chestnut winged cuckoo, Cin Bit: cinnamon bittern, Com Flm: common flameback, Com Ior: common iora, Com Moo: common moorhen, Com Myn: common myna, Com TaB: common tailorbird, Cot PyG: cotton pygmy goose, Dol Bir: dollar bird, Eur AsT: eurasian tree sparrow, Gre Egr: great egret, Gre Cou: greater coucal, Gre Ior: green iora, Gre Her: grey heron, Jun Myn: jungle myna, Lar BiC: large-billed crow, Les Cou: lesser coucal, Les WhD: lesser whistling duck, Lit BrC: little bronze cuckoo, Lit GrP: little green pigeon, Lit Her: little heron, Oli BaS: olive-backed sunbird, Oli WiB: olivewined bulbul, Ora BrP: orange-breasted green pigeon, Ori Mag: oriental magpie robin, Ori ReW: oriental reed warbler, Pac Swa: pacific swallow, Pea Dov: peaceful dove, Phi GIS: Philippine glossy starling, Pie Fan: pied fantail, Pie Tri: pied triller, Pin GrP: pink-necked green pigeon, Pin Sni: pintail snipe, Pla Cuc: plaintive cuckoo, Pur Her: purple heron, Pur SwH: purple swamphen, Red JuF: red junglefowl, Red WaL: red-wattled lapwing, Ric Pip: Richard's pipit, Ruf TaT: rufous-tailed tailorbird, Sav NiJ: savanna nightjar, Sca BrM: scaly-breasted munia, Sch Bit: Schrenck's bittern, Spo Dov: spotted dove, Wat Coc: water cock, Whi BrW: white-breasted waterhen, Whi BrC: white-browed crake, Whi HeM: whiteheaded munia, Whi ThK: white-throated kingfisher, Whi VeM: white-vented myna, Yel Bit: yellow bittern, Yel BeP: yellow-bellied prinia, Yel VeB: yellow-vented bulbul, Zit Cis: zitting cisticola.

\section{Authors' Contribution}

M. N. Rajpar developed the experimental design, conducted research, collected and analyzed data, and drafted paper. M. Zakaria Guided, reviewed and edited the paper.

\section{Acknowledgments}

Authors would like to thank the Department of Wildlife and National Parks, Peninsular Malaysia, for allowing us to conduct this study. This research was partially funded by the Fundamental Grant Research Scheme 01-10-07-291FR and Forestry Sector Research and Development Project, Pakistan, Forest Institute, Peshawar, Pakistan.

\section{References}

[1] J. Burger, "Habitat selection in temperate marsh-nesting birds," in Habitat Selection in Birds, M. L. Cody, Ed., pp. 253281, Academic Press, Orlando, Fla, USA, 1985.

[2] J. Elmberg, P. Nummi, H. Pöysä, and K. Sjöberg, "Relationships between species number, lake size and resource diversity 
in assemblages of breeding waterfowl," Journal of Biogeography, vol. 21, no. 1, pp. 75-84, 1994.

[3] S. Casado and C. Montes, Guía de los Lagosy Humedales de España, J. M. Reyero, Madrid, Spain, 1995.

[4] L. H. Fraser and P. A. Keddy, The World's Largest Wetlands: Ecology and Conservation, Cambridge University Press, Cambridge, UK, 2005.

[5] W. J. Mitsch, "Wetland creation, restoration, and conservation: a wetland invitational at the Olentangy River Wetland Research Park," Ecological Engineering, vol. 24, no. 4, pp. 243251, 2005.

[6] W. J. Mitsch and J. W. Day, "Restoration of wetlands in the Mississippi-Ohio-Missouri (MOM) River Basin: experience and needed research," Ecological Engineering, vol. 26, no. 1, pp. 55-69, 2006.

[7] K. B. Chari, S. A. Abbasi, and S. Ganapathy, "Ecology, habitat and bird community structure at Oussudu lake: towards a strategy for conservation and management," Aquatic Conservation: Marine and Freshwater Ecosystems, vol. 13, no. 5, pp. 373-386, 2003.

[8] M. Fernandez-Aláez, C. Fernandez-Aláez, and S. Rodriguez, "Seasonal changes in biomass of charophytes in shallow lakes in the northwest of Spain," Aquatic Botany, vol. 72, no. 3-4, pp. 335-348, 2002.

[9] B. Altman and J. Bart, "Special Species Monitoring and Assessment in Oregon and Washington: land bird species not adequately monitored by the breeding bird survey," Oregon Washington Partners in Flight, http://www.orwapif.org/pdf/ special_monitoring.pdf, Retrieved on 14th January, 2009.

[10] S. L. Taylor and K. S. Pollard, "Evaluation of two methods to estimate and monitor bird populations," PLoS ONE, vol. 3, no. 8, Article ID e3047, 2008.

[11] W. J. Mitsch, "Wetland Utilization in the World: Protecting Sustainable Use," http://www.globalwetlands.org/Conference Botswana/docs/3\%20Keynotes/Mitsch\%20plenary\%20MS.pdf, Retrieved on 29th April, 2010.

[12] M. W. Weller, Wetland Birds: Habitat Resources and Conservation Implications, Cambridge University Press, Great Britain, UK, 1999.

[13] G. M. Siriwardena, S. R. Baillie, S. T. Buckland, R. M. Fewster, J. H. Marchant, and J. D. Wilson, "Trends in the abundance of farmland birds: a quantitative comparison of smoothed Common Birds Census indices," Journal of Applied Ecology, vol. 35, no. 1, pp. 24-43, 1998.

[14] J. R. Krebs, J. D. Wilson, R .D. Bradbury, and G. M. Siriwardena, "The second silent spring?" Nature, vol. 400, no. 6745, pp. 611-612, 1999.

[15] C. L. Seymour and R. E. Simmons, "Can severely fragmented patches of riparian vegetation still be important for arid-land bird diversity?" Journal of Arid Environments, vol. 72, no. 12, pp. 2275-2281, 2008.

[16] J. A. Veraat, R. S. de Groot, G. Perello, N. J. Riddiford, and R. Roijackers, "Selection of (bio) indicators to assess effects of freshwater use in wetlands: a case study of s'Albufera de Mallorca, Spain," Regional Environmental Change, vol. 4, pp. 107-117, 2004.

[17] Z. Ma, Y. Wang, X. Gan, B. Li, Y. Cai, and J. Chen, "Waterbird population changes in the wetlands at chongming dongtan in the yangtze river estuary, China," Environmental Management, vol. 43, no. 6, pp. 1187-1200, 2009.

[18] R. E. Norvell, F. P. Howe, and J. R. Parrish, "A sevenyear comparison of relative-abundance and distance-sampling methods," Auk, vol. 120, no. 4, pp. 1014-1028, 2003.
[19] J. R. Sauer and W. A. Link, "Hierarchical modeling of population stability and species group attributes from survey data," Ecology, vol. 83, no. 6, pp. 1743-1751, 2002.

[20] K. Jones, Y. Lanthier, P. van der Voet, E. Valkengoed, D. Taylor, and D. Fernández-Prieto, "Monitoring and assessment of wetlands using Earth Observation: the GlobWetland project," Journal of Environmental Management, vol. 90, no. 7, pp. 2154-2169, 2009.

[21] M. R. Kaminski, G. A. Baldassarre, and A. T. Pearse, "Waterbird responses to hydrological management of wetlands reserve program habitats in New York," Wildlife Society Bulletin, vol. 34, no. 4, pp. 921-926, 2006.

[22] K. G. Johnson, M. S. Allen, and K. E. Havens, "A review of littoral vegetation, fisheries, and wildlife responses to hydrologic variation at Lake Okeechobee," Wetlands, vol. 27, no. 1, pp. 110-126, 2007.

[23] H. M. Hagy, G. M. Linz, and W. J. Bleier, "Wildlife conservation sunflower plots and croplands as fall habitat for migratory birds," The American Midland Naturalist, vol. 164, no. 1, pp. 119-135, 2010.

[24] T. R. Mills, M. A. Rumble, and L. D. Flake, "Habitats of birds in ponderosa pine and aspen/birch forest in the Black Hills, South Dakota," Journal of Field Ornithology, vol. 71, no. 2, pp. 187-206, 2000.

[25] J. A. Wiens, The Ecology of the Bird's Communities, Cambridge University Press, Cambridge, UK, 1992.

[26] S. Caziani and E. J. Derlindati, "Abundance and habitat of High Andes Flamingos in Northwestern Argentina," Waterbirds, vol. 23, pp. 121-133, 2000.

[27] M. N. Rajpar and M. Zakaria, "Density and diversity of water birds and terrestrial birds at Paya Indah Wetland Reserve, Selangor Peninsular Malaysia," Journal of Biological Sciences, vol. 10, no. 7, pp. 658-666, 2010.

[28] M. Zakaria, M. N. Rajpar, and S. A. Sajap, "Species diversity and feeding guilds of birds in Paya Indah Wetland Reserve, Peninsular Malaysia," International Journal of Zoological Research, vol. 5, no. 3, pp. 86-100, 2009.

[29] J. Gutzwiller, "Estimating winter species richness with unlimited-distance point counts," Auk, vol. 108, no. 4, pp. 853-862, 1991.

[30] J. E. Jiménez, "Effect of sample size, plot size, and counting time on estimates of avain diversity and abundance in a Chilean rainforest," Journal of Field Ornithology, vol. 71, no. 1, pp. 66-87, 2000.

[31] D. C. Lee and S. J. Marsden, "Adjusting count period strategies to improve the accuracy of forest bird abundance estimates from point transect distance sampling surveys," Ibis, vol. 150, no. 2, pp. 315-325, 2008.

[32] C. J. Bibby, N. D. Burgess, D. A. Hill, and S. Mustoe, Bird Census Techniques, Academic Press, London, UK, 2nd edition, 2000.

[33] S. T. Buckland, D. R. Anderson, K. P. Burnhan, J. L. Lake, D. L. Borchers, and L. Thomas, Advance Distance Sampling; Estimating Abundance of Biological Populations, Campman and Hall, London, UK, 2004.

[34] D. A. Aborn, "Abundance, density, and diversity of neotropical migrants at the Lula Lake Land Trust, GA," Southeastern Naturalist, vol. 6, no. 2, pp. 293-304, 2007.

[35] C. P. Nadeau, C. J. Conway, B. S. Smith, and T. E. Lewis, "Maximizing detection probability of Wetland-dependent birds during point-count surveys in northwestern Florida," Wilson Journal of Ornithology, vol. 120, no. 3, pp. 513-518, 2008.

[36] P. J. Mumby, A. J. Edwards, E. P. Green, C. W. Anderson, A. C. Ellis, and C. D. Clark, "A visual assessment technique 
for estimating seagrass standing crop," Aquatic Conservation: Marine and Freshwater Ecosystems, vol. 7, no. 3, pp. 239-251, 1997.

[37] C. Hudon, "Impact of water level fluctuations on St. Lawrence River aquatic vegetation," Canadian Journal of Fisheries and Aquatic Sciences, vol. 54, no. 12, pp. 2853-2865, 1997.

[38] J. P. Isacch, N. O. Maceira, M. S. Bo, M. R. Demaría, and S. Peluc, "Bird-habitat relationship in semi-arid natural grasslands and exotic pastures in the west pampas of Argentina," Journal of Arid Environments, vol. 62, no. 2, pp. 267-283, 2005.

[39] C. J. F. Ter Braak and P. Smilauer, Canoco Reference Manual and Canoco Draw for Windows User's Guide: Software for Canonical Ordination (Version 4.5), Ithaca, NY, USA, 2002.

[40] M. Brown and J. J. Dinsmore, "Implications of marsh size and isolation for marsh bird management," Journal of Wildlife Management, vol. 50, no. 3, pp. 392-397, 1986.

[41] S. E. Fairbairn and J. J. Dinsmore, "Factors associated with occurrence and density of wetland birds in the Prairie Pothole region of Iowa," Journal of the Iowa Academy of Science, vol. 108, no. 1, pp. 8-14, 2001.

[42] S. L. Pimm and M. L. Rosenzweig, "Competitors and habitat use," Oikos, vol. 37, pp. 1-6, 1981.

[43] M. L. Rosenzweig, "Some theoretical aspects of habitat selection," in Habitat Selection in Birds, M. L. Cody, Ed., pp. 517540, Academic Press, New York, NY, USA, 1985.

[44] T. E. Martin, "Life history evolution in tropical and south temperate birds: what do we really know?" Journal of Avian Biology, vol. 27, no. 4, pp. 263-272, 1996.

[45] W. F. Masifwa, T. Twongo, and P. Denny, "The impact of water hyacinth, Eichhornia crassipes (Mart) solms on the abundance and diversity of aquatic macroinvertebrates along the shores of northern Lake Victoria, Uganda," Hydrobiologia, vol. 452, no. 1-3, pp. 79-88, 2001.

[46] J. D. Toft, C. A. Simenstad, J. R. Cordell, and L. F. Grimaldo, "The effects of introduced water hyacinth on habitat structure, invertebrate assemblages, and fish diets," Estuaries, vol. 26, no. 3, pp. 746-758, 2003.

[47] M. Meerhoff, N. Mazzeo, B. Moss, and L. Rodríguez-Gallego, "The structuring role of free-floating versus submerged plants in a subtropical shallow lake," Aquatic Ecology, vol. 37, no. 4, pp. 377-391, 2003.

[48] R. M. Kaminiski and H. H. Prince, "Dabbling duck-habitat association during spring in Delta Marsh, Manitoba," Journal of Wildlife Management, vol. 10, no. 1, pp. 37-50, 1984.

[49] P. Nummi, H. Pdysä, J. Elmberg, and K. Sjöberg, "Habitat distribution of the mallard in relation to vegetation structure, food, and population density," Hydrobiologia, vol. 279-280, no. 1, pp. 247-252, 1994.

[50] S. F. Mitchell and M. R. Perrow, "Interactions between grazing birds and macrophytes," in The Structuring Role of Submerged Macrophytes in Lakes, E. Jeppesen, M. Søndergaard, and K. Christoffersen, Eds., vol. 131, pp. 175-196, Springer, New York, NY, USA, 1998.

[51] R. Noordhuis, D. T. van der Molen, and M. S. van den Berg, "Response of herbivorous water-birds to the return of Chara in Lake Veluwemeer, The Netherlands," Aquatic Botany, vol. 72, no. 3-4, pp. 349-367, 2002.

[52] S. R. Carpenter and D. M. Lodge, "Effects of submersed macrophytes on ecosystem processes," Aquatic Botany, vol. 26, no. C, pp. 341-379, 1986.

[53] A. Prejs, "Herbivory by temperate freshwater fishes and its consequences," Environmental Biology of Fishes, vol. 10, no. 4, pp. 281-296, 1984.
[54] D. M. Lodge, "Herbivory on freshwater macrophytes," Aquatic Botany, vol. 41, no. 1-3, pp. 195-224, 1991.

[55] E. Jeppesen, M. Søndergaard, M. Søndergaard, and K. Christoffersen, The Structuring Role of Submerged Macrophytes in Lakes. Ecological Studies, vol. 131, Springer, New York, NY, USA, 1998.

[56] K. L. van Rees-Siewert and J. J. Dinsmore, "Influence of wetland age on bird use of restored wetlands in Iowa," Wetlands, vol. 16, no. 4, pp. 577-582, 1996.

[57] R. S. King and D. A. Wrubleski, "Spatial and diel availability of flying insects as potential duckling food in prairie wetlands," Wetlands, vol. 18, no. 1, pp. 100-114, 1998.

[58] L. K. Benoit and R. A. Askins, "Impact of the spread of Phragmites on the distribution of birds in Connecticut tidal marshes," Wetlands, vol. 19, no. 1, pp. 194-208, 1999.

[59] G. I. M. Linz, D. C. Blixt, D. L. Bergman, and W. J. Bleier, "Responses of Red-winged Blackbirds, Yellow-headed Blackbirds and Marsh Wrens to glyphosate-induced alterations in cattail density," Journal of Field Ornithology, vol. 67, no. 1, pp. 167-176, 1996.

[60] G. M. Linz, D. L. Bergman, D. C. Blixr, and W.J. Bleier, "Black Terns benefit from cattail management in the northern Great Plains," Colonial Waterbirds, vol. 20, pp. 617-621, 1997.

[61] J. A. Surdick Jr., Biotic and abiotic indicators of foraging site selection and foraging success of four ciconiiform species in the freshwater Everglades of Florida, M.S. thesis, University of Florida, Gainesville, Fla, USA, 1998.

[62] R. J. Safran, C. R. Isola, M. A. Colwell, and O. E. Williams, "Benthic invertebrates at foraging locations of nine waterbird species in managed wetlands of the northern San Joaquin Valley, California," Wetlands, vol. 17, no. 3, pp. 407-415, 1997.

[63] G. Katzir, T. Strod, E. Schechtman, S. Hareli, and Z. Arad, "Cattle egrets are less able to cope with light refraction than are other herons," Animal Behaviour, vol. 57, no. 3, pp. 687694, 1999.

[64] J. Hancock and J. Kushlan, The Herons Handbook, Croom Helm, London, UK, 1984.

[65] A. Lotem, E. Schechtman, and G. Katzir, "Capture of submerged prey by little egrets, Egretta garzetta garzetta: strike depth, strike angle and the problem of light refraction," Animal Behaviour, vol. 42, no. 3, pp. 341-346, 1991.

[66] J. C. Z. Woinarski, S. C. Tidemann, and S. Kerin, "Birds in a tropical mosaic: the distribution of bird species in relation to vegetation patterns," Australian Wildlife Research, vol. 15, no. 2, pp. 171-196, 1988.

[67] S. H. Anderson, H. H. Shugart Jr., and T. M. Smith, "Vertical and temporal habiotat utilization within a breeding bird community," in The Role of Insectivorous Birds in Forest Ecosystems, J. G. Dickson, R. N. Conner, R. R. Fleet, J. C. Kroll, and J. A. Jackson, Eds., pp. 261-268, Academic Press, New York, NY, USA, 1979.

[68] R. C. Macnally, "The roles of floristics and physiognomy in avian community composition," Australian Journal of Ecology, vol. 15, no. 3, pp. 321-327, 1990.

[69] M. E. Harmon, J. F. Franklin, F. J. Swanson et al., "Ecology of Coarse Woody Debris in Temperate Ecosystems," Advances in Ecological Research, vol. 15, no. C, pp. 133-302, 1986.

[70] R. B. Huey, E. R. Pianka, and T. W. Schoener, Lizard Ecology. Studies of a Model Organism, Harvard University Press, Cambridge, Mass, USA, 1993.

[71] J. T. Rotenberry, "The role of habitat in avian community composition: physiognomy or floristics?" Oecologia, vol. 67, no. 2, pp. 213-217, 1985. 
[72] W. M. Block and L. A. Brennan, "The habitat concept in ornithology: theory and applications," Current Ornithology, vol. 11, pp. 35-89, 1999.

[73] M. G. Yates, J. D. Goss-Custard, S. McGrorty et al., "Sediment characteristics, invertebrate densities and shorebird densities on the inner banks of the Wash," Journal of Applied Ecology, vol. 30, no. 4, pp. 599-614, 1993.

[74] Y. Zharikov and G. A. Skilleter, "Sex-specific intertidal habitat use in subtropically wintering Bar-tailed Godwits," Canadian Journal of Zoology, vol. 80, no. 11, pp. 1918-1929, 2002.

[75] T. B. Champlin, J. C. Kilgo, M. L. Gumpertz, and C. E. Moorman, "Avian response to microclimate in canopy gaps in a bottomland hardwood forest," Southeastern Naturalist, vol. 8, no. 1, pp. 107-120, 2009.

[76] J. R. Karr and K. E. Freemark, "Habitat selection and environmental gradients: dynamics in the "stable" tropics," Ecology, vol. 64, no. 6, pp. 1481-1494, 1983.

[77] T. E. Martin and C. K. Ghalambor, "Males feeding females during incubation. I. Required by microclimate or constrained by nest predation?" American Naturalist, vol. 153, no. 1, pp. 131-139, 1999.

[78] D. G. Wachob, "The effect of thermal microclimate on foraging site selection by wintering mountain chickadees," Condor, vol. 98, no. 1, pp. 114-122, 1996.

[79] G. E. Walsberg, "Physiological consequences of microhabitat selection," in Habitat Selection in Birds, M. L. Cody, Ed., p. 558, Academic Press, New York, NY, USA, 1985.

[80] J. Chen, J. F. Franklin, and T. A. Spies, "Contrasting microclimates among clearcut, edge, and interior of old-growth Douglas-fir forest," Agricultural and Forest Meteorology, vol. 63, no. 3-4, pp. 219-237, 1993.

[81] R. DeWoskin, "Heat exchange influence on foraging behavior in Zonotrichia flocks," Ecology, vol. 61, pp. 30-36, 1980.

[82] J. A. Kushlan, "Resource use strategies in wading birds," Wilson Bulletin, vol. 93, pp. 145-163, 1981.

[83] R. M. Erwin, "Feeding habitats of nesting wading birds: spatial use and social influences," Auk, vol. 100, no. 4, pp. 960-970, 1983.

[84] R. W. Butler and R. G. Vennesland, "Integrating climate change and predation risk with wading bird conservation research in North America," Colonial Waterbirds, vol. 23, no. 3, pp. 535-540, 2000.

[85] J. W. Rivers, "Northern Harrier predation of White-faced Ibis," Wilson Bulletin, vol. 112, no. 3, pp. 416-417, 2000. 

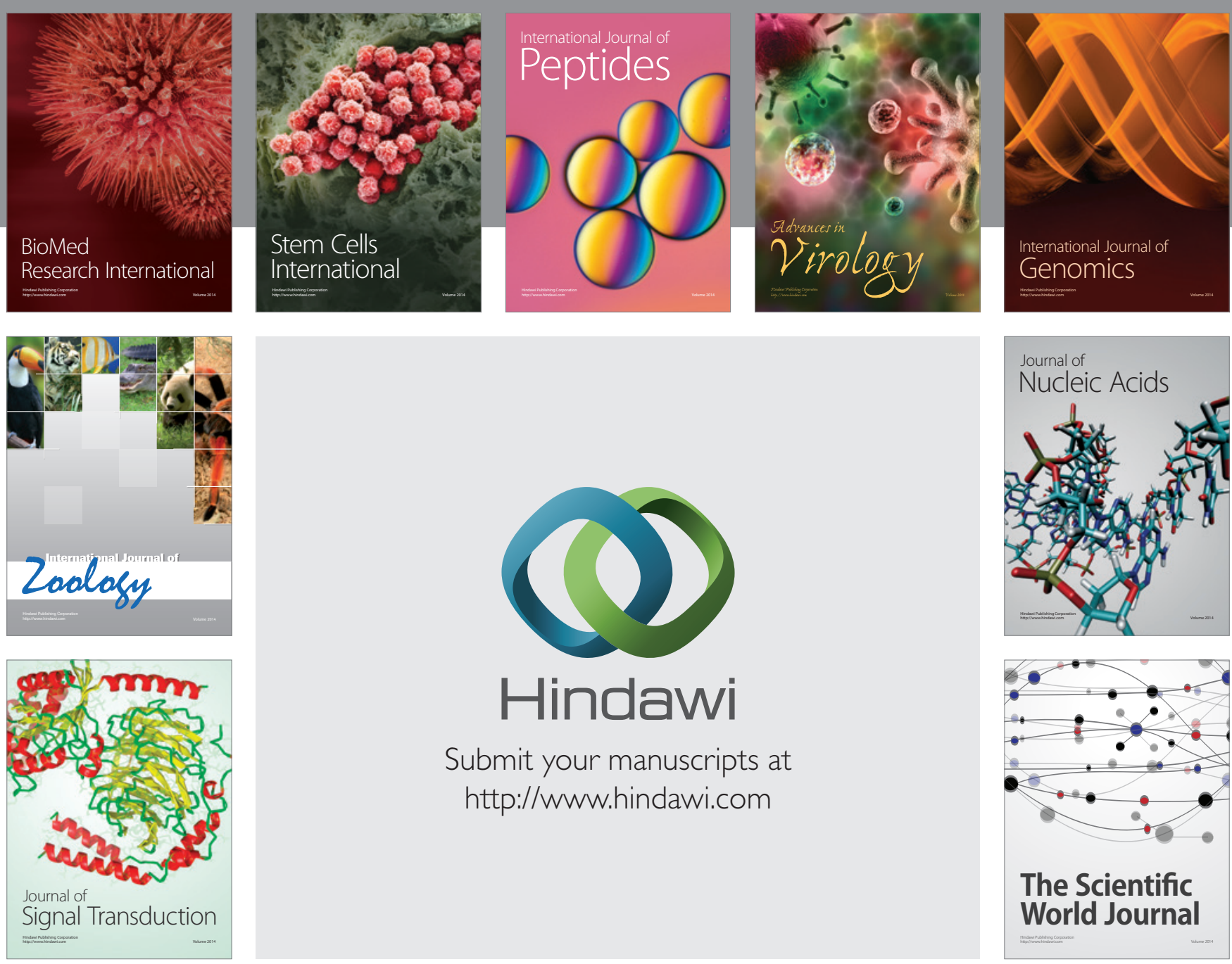

Submit your manuscripts at

http://www.hindawi.com
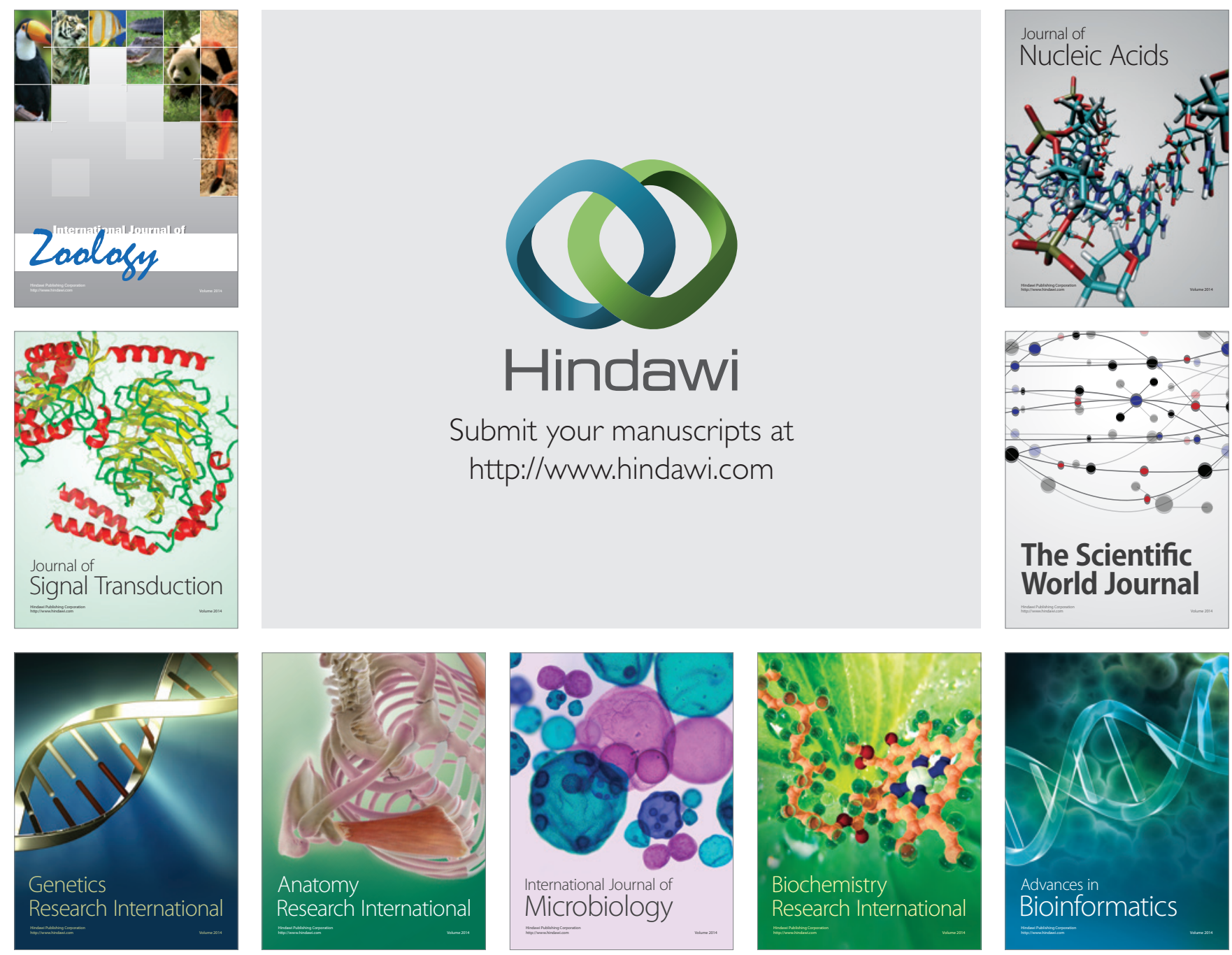

The Scientific World Journal
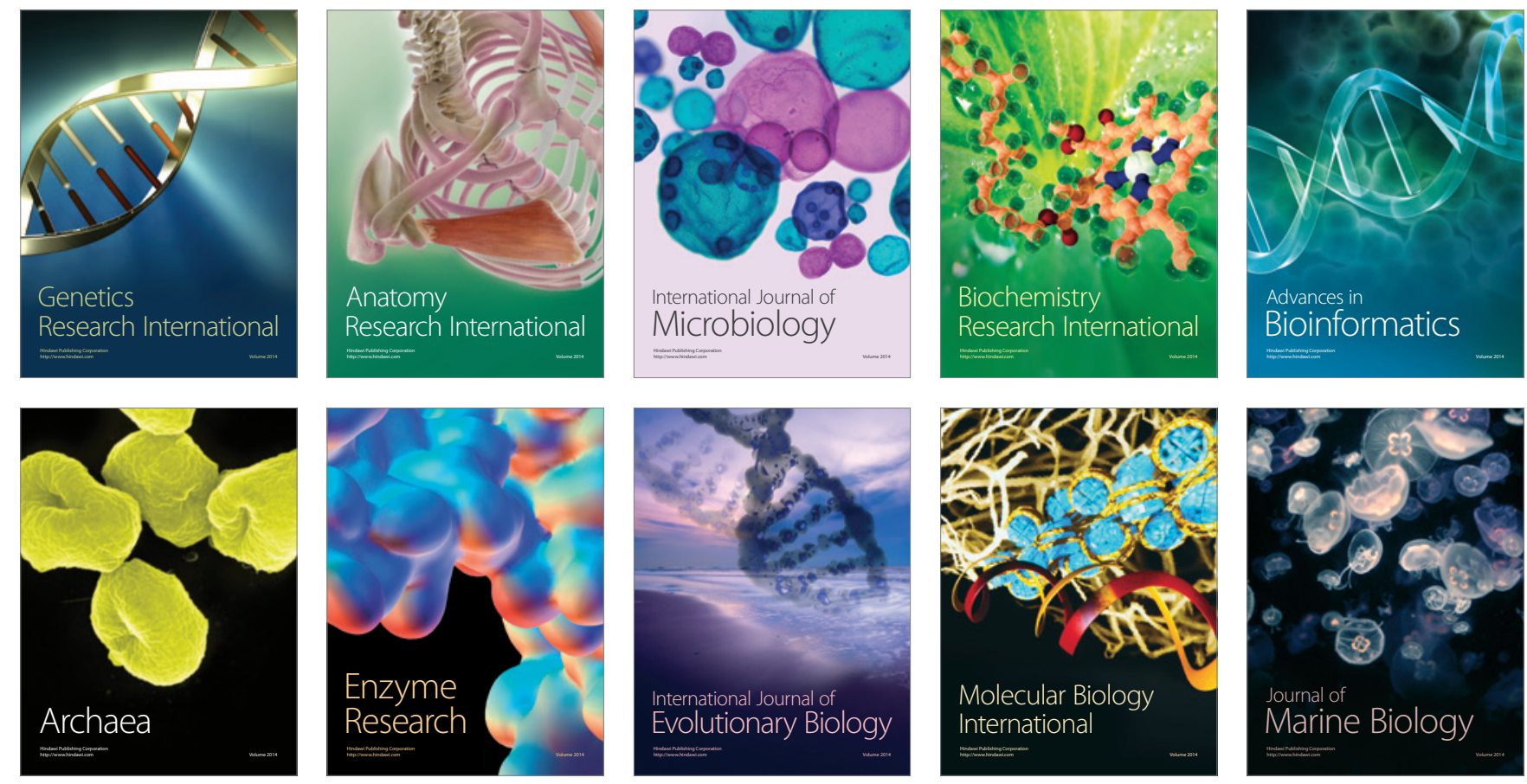